\title{
Quantum master equation for collisional dynamics of massive particles with internal degrees of freedom
}

\author{
Andrea Smirne* and Bassano Vacchini ${ }^{\dagger}$ \\ Universitá degli Studi di Milano, Dipartimento di Fisica, Via Celoria 16, I-20133 Milano, Italy and \\ INFN, Sezione di Milano, Via Celoria 16, I-20133 Milano, Italy
}

(Received 1 March 2010; published 25 October 2010)

\begin{abstract}
We address the microscopic derivation of a quantum master equation in Lindblad form for the dynamics of a massive test particle with internal degrees of freedom, interacting through collisions with a background ideal gas. When either internal or center-of-mass degrees of freedom can be treated classically, previously established equations are obtained as special cases. If in an interferometric setup the internal degrees of freedom are not detected at the output, the equation can be recast in the form of a generalized Lindblad structure, which describes non-Markovian effects. The effect of internal degrees of freedom on center-of-mass decoherence is considered in this framework.
\end{abstract}

DOI: 10.1103/PhysRevA.82.042111

PACS number(s): 03.65.Yz, 05.20.Dd, 03.75.-b, 03.65.Ta

\section{INTRODUCTION}

In recent times, major advances in experimental techniques have led to the realization of experiments in which quantum systems in a single-particle regime are studied under their controlled interaction with some environment. A paradigmatic example in this context is given by the motion of a massive test particle in an interferometric setup, which gives rise to interference fringes as typical quantum signatures. When coupling with the environment becomes relevant, such interference fringes are gradually washed out, and a classical dynamics is eventually recovered. This phenomenon goes by the name of decoherence [1-3]. Its understanding and theoretical description require, on the one hand, a control over the environment, and, on the other hand, a microscopic model for the interaction and the ensuing dynamics.

For the case of a tracer particle immersed in a dilute gas, such a microscopic description has been obtained considering the center-of-mass degrees of freedom only. The reduced dynamics is given by a master equation in Lindblad form which has been called the quantum linear Boltzmann equation, since it provides the natural quantum counterpart of the classical linear Boltzmann equation (see [4] for a recent review and references therein). The microscopic input is given by the complex scattering amplitudes describing the collisions between gas and test particle, while the gas is characterized by its density and momentum distribution. In this paper, we consider an extension of this result, which includes internal degrees of freedom of the tracer particle. The microscopic derivation is performed along the lines of a general strategy for the derivation of Markovian master equations, which relies on a scattering description of the interaction events [5]. Besides the gas properties, this approach takes as basic input the multichannel complex scattering amplitudes, which describe the influence of the internal states on the scattering events. Indeed, when the scattering cross section depends not only on

\footnotetext{
*andrea.smirne@unimi.it

†bassano.vacchini@mi.infn.it
}

the relative motional state between tracer and gas particle, such an extension becomes mandatory in order to correctly describe the dynamics. According to the Markovian approximation, the obtained master equation is in Lindblad form. This derivation confirms the structure of the dissipative term, which was heuristically obtained in [6], further determining the coherent contribution to the dynamics due to forward scattering. The latter becomes relevant in the determination of the index of refraction for matter waves. When either type of degrees of freedom can be described in classical terms, a Markovian quantum classical master equation is obtained. Such a result, corresponding to a classical treatment of the motional degrees of freedom, was considered in [7]. In that context, the name Bloch-Boltzmann was proposed for the equation, since for a two-level system an extension of the optical Bloch equations to include a Boltzmann-like collision term is obtained. In the same spirit, the name quantum Bloch-Boltzmann can be used to indicate a master equation, which gives a quantum description of both internal and center-of-mass states.

An interesting situation appears when in the final detection the internal state of the test particle is not resolved at the output of the interferometer. In this case, the internal degrees of freedom become part of the environment. Then a nonMarkovian dynamics for the motional state appears, which can be described in terms of a coupled set of Lindblad equations for the unnormalized statistical operators corresponding to specific internal channels. This type of non-Markovian dynamics can be considered a generalized non-Markovian Lindblad structure. It arises as a mean over a classical index, which can take place, for examples, as a consequence of the interaction with a structured reservoir $[6,8,9]$. This situation is considered here in the study of the loss of visibility of the interference fringes in an interferometric setup. The ensuing decoherence effect is generally not described as an exponential loss of visibility depending on the strength of the interaction, as in the usual Markovian case.

The paper is organized as follows. In Sec. II, we consider the expression of the master equation, pointing to the main steps necessary for its derivation and putting into evidence the microscopic quantities determining its explicit form. 
A detailed microscopic derivation of the master equation is performed in Appendix. The master equation is given in terms of matrix elements of the statistical operator in the momentum and internal energy eigenstates basis, as well as an explicit operator expression, which makes its Lindblad structure manifest. This also allows us to easily recover under suitable limits previously considered master equations, which describe either only one of the two kind of degrees of freedom or a hybrid quantum classical description of both. In Sec. III, we show how the interplay between internal and motional states can influence the visibility in an interferometric setup for the study of decoherence, leading to non-Markovian behavior in the reduction of the visibility of the interference fringes.

\section{THE MASTER EQUATION FOR A TEST PARTICLE WITH INTERNAL DEGREES OF FREEDOM}

We first consider the key ingredients and steps that lead to obtaining the master equation describing the collisional dynamics of a test particle immersed in a structureless background gas, taking the internal degrees of freedom of the particle into account. The task of a full microscopic derivation is accomplished in Appendix, relying on a method recently introduced for the derivation of Markovian master equations, which has been called the monitoring approach [5,10-12]. In the monitoring approach, the reduced dynamics of a system in contact with some environment is obtained describing the interaction by means of scattering theory. The building blocks in such a formulation of the open system dynamics are therefore the $S$ matrix characterizing the single-interaction events and the rate of collisions. Both quantities are given by operators on the tensor-product Hilbert space of system and environment, which we denote by $\mathrm{S}=\mathrm{I}+i \mathrm{~T}$ and $\Gamma$, respectively. The operator nature of these quantities is crucial in order to take the gas and test-particle state into account in the dynamic description of the collisional interaction. The Markovian master equation for the reduced dynamics is obtained by assuming the various collisions are independent, so their effect cumulates according to the state-dependent scattering rate, and taking the trace over the environmental degrees of freedom.

\section{A. Expression of the master equation}

The formal expression of the master equation reads [5]

$$
\frac{d}{d t} \rho=\frac{1}{i \hbar}[H, \rho]+\mathcal{L} \rho+\mathcal{R} \rho,
$$

where $H$ is the free Hamiltonian and $\rho$ is the statistical operator of the system. For the case at hand, the free Hamiltonian of the system is given by

$$
H=\frac{\mathrm{P}^{2}}{2 M} \otimes \sum_{i} \hbar \omega_{i}|i\rangle\langle i|,
$$

where $\mathrm{P}$ is the momentum operator of the test particle, $M$ is its mass, and $\{|i\rangle\}_{i=1, \ldots, n}$ is the basis of energy eigenstates in $\mathbb{C}^{n}$. The superoperators appearing on the right-hand side (rhs) of Eq. (1) are defined according to

$$
\begin{aligned}
\mathcal{L} \rho= & \operatorname{Tr}_{\text {gas }}\left(\mathrm{T} \Gamma^{1 / 2}\left[\rho \otimes \rho_{\text {gas }}\right] \Gamma^{1 / 2} \mathrm{~T}^{\dagger}\right) \\
& -\frac{1}{2} \operatorname{Tr}_{\text {gas }}\left(\Gamma^{1 / 2} \mathrm{~T}^{\dagger} \mathrm{T} \Gamma^{1 / 2}\left[\rho \otimes \rho_{\text {gas }}\right]\right) \\
& -\frac{1}{2} \operatorname{Tr}_{\text {gas }}\left(\left[\rho \otimes \rho_{\text {gas }}\right] \Gamma^{1 / 2} \mathrm{~T}^{\dagger} \mathrm{T} \Gamma^{1 / 2}\right)
\end{aligned}
$$

and

$$
\mathcal{R} \rho=i \operatorname{Tr}_{\text {gas }}\left\{\left[\Gamma^{1 / 2} \operatorname{Re}(\mathrm{T}) \Gamma^{1 / 2}, \rho \otimes \rho_{\text {gas }}\right]\right\},
$$

respectively, where $\rho_{\text {gas }}$ is the single-particle statistical operator describing the gas environment. Note that the operators $\mathcal{L}$ and $\mathcal{R}$ arise by acting with an operator in Lindblad form on a state of system plus gas in factorized form, further taking the partial trace with respect to the gas. While this operation is formally legitimate and guarantees preservation of trace and hermiticity of the statistical operator describing the test particle, it is generally not true that the resulting dynamics for the reduced system only is given by a master equation in Lindblad form, thus granting complete positivity and describing a well-defined Markovian dynamics. Indeed this step involves further approximations, which depend in a crucial way on details of the system and interaction. It is well known that by taking the partial trace with respect to the unitary evolution of the overall system one can obtain a Markovian dynamics only if further hypotheses hold. This remains true for the case at hand, despite the fact that important approximations have already been introduced in replacing the Hamiltonian dynamics for system plus gas with a Lindblad operator only specified by $\mathrm{T}$ and $\Gamma$. The actual proof that a Markovian dynamics applies to the situation of interest and the specific expression of the superoperators appearing in Eq. (1) is obtained through the microscopic calculations performed in Appendix.

Relying on the results of Appendix 1a, we write the following expression for the contributions in Eq. (3) in the momentum and channel basis $\{|\boldsymbol{P}, i\rangle\}$ :

$$
\begin{aligned}
\left\langle\boldsymbol{P}, i|\mathcal{L} \rho| \boldsymbol{P}^{\prime}, k\right\rangle & \\
= & \sum_{j l} \int d \boldsymbol{Q}\left[\left\langle\boldsymbol{P}-\boldsymbol{Q}, j|\rho| \boldsymbol{P}^{\prime}-\boldsymbol{Q}, l\right\rangle M_{i k}^{j l}\left(\boldsymbol{P}, \boldsymbol{P}^{\prime} ; \boldsymbol{Q}\right)\right. \\
& -\frac{1}{2}\left\langle\boldsymbol{P}, j|\rho| \boldsymbol{P}^{\prime}, k\right\rangle M_{l l}^{j i}(\boldsymbol{P}+\boldsymbol{Q}, \boldsymbol{P}+\boldsymbol{Q} ; \boldsymbol{Q}) \\
& \left.-\frac{1}{2}\left\langle\boldsymbol{P}, i|\rho| \boldsymbol{P}^{\prime}, l\right\rangle M_{j j}^{k l}\left(\boldsymbol{P}^{\prime}+\boldsymbol{Q}, \boldsymbol{P}^{\prime}+\boldsymbol{Q} ; \boldsymbol{Q}\right)\right],
\end{aligned}
$$

where the complex rate functions $M_{i k}^{j l}\left(\boldsymbol{P}, \boldsymbol{P}^{\prime} ; \boldsymbol{Q}\right)$ are given by

$$
\begin{aligned}
& M_{i k}^{j l}\left(\boldsymbol{P}, \boldsymbol{P}^{\prime} ; \boldsymbol{Q}\right) \\
& \quad=\chi_{i k}^{j l} \int_{\boldsymbol{Q}_{\perp}} d \boldsymbol{p} L_{i j}(\boldsymbol{p}, \boldsymbol{P}-\boldsymbol{Q} ; \boldsymbol{Q}) L_{k l}^{*}\left(\boldsymbol{p}, \boldsymbol{P}^{\prime}-\boldsymbol{Q} ; \boldsymbol{Q}\right),
\end{aligned}
$$

and $\chi_{i k}^{j l}$ is a notational shorthand to indicate that the contribution is different from zero only for $\mathcal{E}_{i j}=\mathcal{E}_{k l}$, where $\mathcal{E}_{k j}=E_{k}-E_{j}$ denotes the difference in energy between internal states, while the $\boldsymbol{p}$ integration is restricted to the plane $\boldsymbol{Q}_{\perp}=\left\{\boldsymbol{p} \in \mathbb{R}^{3}: \boldsymbol{p} \cdot \boldsymbol{Q}=0\right\}$. The functions $L_{i j}(\boldsymbol{p}, \boldsymbol{P} ; \boldsymbol{Q})$ are 
defined according to

$$
\begin{aligned}
& L_{i j}(\boldsymbol{p}, \boldsymbol{P} ; \boldsymbol{Q}) \sqrt{\frac{n_{\mathrm{gas}} m}{m_{*}^{2} Q}} \sqrt{\mu\left[\boldsymbol{p}_{\perp}+\frac{m}{M} \boldsymbol{P}_{\|}+\left(1+\frac{m}{M}\right) \frac{\boldsymbol{Q}}{2}+\frac{\mathcal{E}_{i j}}{Q^{2} / m} \boldsymbol{Q}\right]} \\
& \quad \times f_{i j}\left(\operatorname{rel}\left(\boldsymbol{p}_{\perp}, \boldsymbol{P}_{\perp}\right)-\frac{\boldsymbol{Q}}{2}+\frac{\mathcal{E}_{i j}}{Q^{2} / m_{*}} \boldsymbol{Q}\right. \\
&\left.\quad \operatorname{rel}\left(\boldsymbol{p}_{\perp}, \boldsymbol{P}_{\perp}\right)+\frac{\boldsymbol{Q}}{2}+\frac{\mathcal{E}_{i j}}{Q^{2} / m_{*}} \boldsymbol{Q}\right)
\end{aligned}
$$

where $\mu(\boldsymbol{p})$ denotes the stationary gas distribution, $n_{\mathrm{gas}}$ is the density of the gas, $m$ is the mass of the gas particles, $m_{*}=$ $m M /(M+m)$ is the reduced mass, and $f_{k j}\left(\boldsymbol{p}_{f}, \boldsymbol{p}_{i}\right)$ denotes the multichannel complex scattering amplitudes, which depend on the microscopic interaction potential and describe scattering from an initial momentum $\boldsymbol{p}_{i}$ and internal state $j$ to a final state with momentum $\boldsymbol{p}_{f}$ and internal state $k$. Moreover, $\boldsymbol{P}_{\perp}$ and $\boldsymbol{P}_{\|}$indicate the perpendicular and parallel components, respectively, of the momentum $\boldsymbol{P}$ with respect to the vector $\boldsymbol{Q}$; $\operatorname{rel}(\boldsymbol{p}, \boldsymbol{P}) \equiv\left(m_{*} / m\right) \boldsymbol{p}-\left(m_{*} / \boldsymbol{M}\right) \boldsymbol{P}$ is the relative momentum between the gas-particle momentum $\boldsymbol{p}$ and the test-particle momentum $\boldsymbol{P}$.

Exploiting these results, we can easily write the master equation, Eq. (1), directly in operator form. In fact, using the functions $L_{i j}(\boldsymbol{p}, \boldsymbol{P} ; \boldsymbol{Q})$, let us introduce the following family of jump operators:

$$
\mathrm{L}_{\boldsymbol{Q}, \boldsymbol{p}, \mathcal{E}}=\mathrm{e}^{i \boldsymbol{Q} \cdot \mathrm{X} / \hbar} \sum_{\substack{i j \\ \mathcal{E}_{i j}=\mathcal{E}}} L_{i j}(\boldsymbol{p}, \mathrm{P} ; \boldsymbol{Q}) \otimes \mathrm{E}_{i j},
$$

where $\mathrm{X}$ and $\mathrm{P}$ are position and momentum operators of the test particle. The operators $\mathrm{E}_{i j}=|i\rangle\langle j|$ act on the internal degrees of freedom only, since $|i\rangle$ denotes the energy eigenstate with eigenvalue $\hbar \omega_{i}$, and the exponential factor describes momentum exchanges according to $\exp (i \mathbf{X} \cdot \boldsymbol{Q} / \hbar)|\boldsymbol{P}\rangle=|\boldsymbol{P}+\boldsymbol{Q}\rangle$. Note that the functions $L_{i j}(\boldsymbol{p}, \boldsymbol{P} ; \boldsymbol{Q})$ essentially depend on the scattering amplitudes and the momentum distribution of the gas, thus taking into account all the details of the collisional interaction. These expressions appear operator-valued in the master equation, being evaluated for $\boldsymbol{P} \rightarrow \mathrm{P}$, so as to take into account the actual momentum of the colliding test particle. The incoherent contribution $\mathcal{L}$ in Eq. (1) finally reads

$$
\begin{aligned}
\mathcal{L} \rho= & \sum_{\mathcal{E}} \int d \boldsymbol{Q} \int_{\boldsymbol{Q}_{\perp}} d \boldsymbol{p} \\
& \times\left(\mathrm{L}_{\boldsymbol{Q}, \boldsymbol{p}, \mathcal{E}} \rho \mathrm{L}_{\boldsymbol{Q}, \boldsymbol{p}, \mathcal{E}}^{\dagger}-\frac{1}{2}\left\{\mathrm{~L}_{\boldsymbol{Q}, \boldsymbol{p}, \mathcal{E}}^{\dagger} \mathrm{L}_{\boldsymbol{Q}, \boldsymbol{p}, \mathcal{E}, \rho\}}\right) .\right.
\end{aligned}
$$

The superoperator $\mathcal{R}$ of Eq. (4) on its turn according to Appendix 1b amounts to the commutator with an effective Hamiltonian given by

$$
\begin{aligned}
H_{n}= & -2 \pi \hbar^{2} \frac{n_{\text {gas }}}{m_{*}} \sum_{\substack{i j \\
\mathcal{E}_{i j}=0}} \int d \boldsymbol{p}_{0} \mu\left(\boldsymbol{p}_{0}\right) \\
& \times \operatorname{Re}\left[f_{i j}\left(\operatorname{rel}\left(\boldsymbol{p}_{0}, \mathrm{P}\right), \operatorname{rel}\left(\boldsymbol{p}_{0}, \mathrm{P}\right)\right)\right] \otimes \mathrm{E}_{i j} .
\end{aligned}
$$

Using the alternative expression given by Eq. (A18) to define $L_{i j}(\boldsymbol{p}, \boldsymbol{P} ; \boldsymbol{Q})$, it is immediately seen that the incoherent term of this master equation confirms the result heuristically obtained in [6]. In the latter reference, this equation was also termed the quantum Bloch-Boltzmann equation in that it provides a quantum description of both motional and internal degrees of freedom, thus extending the result of [13], where the center-ofmass degrees of freedom were treated classically and the name Bloch-Boltzmann equation was used. These names should not confuse the reader. Indeed, only for the case of an atom in a two-level approximation undergoing collisional dynamics does this equation refer to an extension of the optical Bloch equations with a Boltzmann collision term.

\section{B. Limiting forms}

As a compatibility check of the master equation derived in Appendix, and in order to make contact with previous work, we now show how in suitable limits it recovers already-known equations. Since the equation describes the quantum dynamics of a test particle with both internal and translational degrees of freedom immersed in a dilute gas, natural limiting situations appear when we consider a structureless test particle or an immobile system. These situations correspond to the quantum linear Boltzmann equation [12] and to the master equation for an immobile system interacting through collisions with a background gas $[5,14]$. Another natural limit consists of a hybrid quantum classical description, in which either internal or center-of-mass degrees of freedom are treated classically. The master equation corresponding to this last case was already considered in [13]. A classical treatment of both kinds of degrees of freedom leads to the master equation for a classical Markov process, with a probability density depending on both a discrete and a continuous index.

\section{Quantum linear Boltzmann equation}

If the internal degrees of freedom can be disregarded, the sum in Eq. (9) has a single nonvanishing contribution, so instead of the multichannel scattering amplitudes $f_{i j}\left(\boldsymbol{p}_{f}, \boldsymbol{p}_{i}\right)$ there is a single amplitude which can be indicated as $f\left(\boldsymbol{p}_{f}, \boldsymbol{p}_{i}\right)$. The incoherent term in the master equation reduces to

$$
\mathcal{L} \rho=\int d \boldsymbol{Q} \int_{\boldsymbol{Q}_{\perp}} d \boldsymbol{p}\left(\mathrm{L}_{\boldsymbol{Q}, \boldsymbol{p}} \rho \mathrm{L}_{\boldsymbol{Q}, \boldsymbol{p}}^{\dagger}-\frac{1}{2}\left\{\mathrm{~L}_{\boldsymbol{Q}, \boldsymbol{p}}^{\dagger} \mathrm{L}_{\boldsymbol{Q}, \boldsymbol{p}}, \rho\right\}\right)
$$

with

$$
\begin{aligned}
\mathrm{L}_{\boldsymbol{Q}, \boldsymbol{p}}= & \sqrt{\frac{n_{\mathrm{gas}} m}{m_{*}^{2} Q}} \mathrm{e}^{i \boldsymbol{Q} \cdot \mathrm{X} / \hbar} \sqrt{\mu\left(\boldsymbol{p}_{\perp}+\frac{m}{M} \mathrm{P}_{\|}+\left(1+\frac{m}{M}\right) \frac{\boldsymbol{Q}}{2}\right)} \\
& \times f\left(\operatorname{rel}\left(\boldsymbol{p}_{\perp}, \mathrm{P}_{\perp}\right)-\frac{\boldsymbol{Q}}{2}, \operatorname{rel}\left(\boldsymbol{p}_{\perp}, \mathrm{P}_{\perp}\right)+\frac{\boldsymbol{Q}}{2}\right),
\end{aligned}
$$

while the Hamiltonian term reads $H_{0}+H_{n}$, where $H_{0}=$ $\mathrm{P}^{2} / 2 M$ and

$$
H_{n}=-\frac{2 \pi \hbar^{2} n_{\text {gas }}}{m_{*}} \int d \boldsymbol{p}_{0} \mu\left(\boldsymbol{p}_{0}\right) \operatorname{Re}\left[f\left(\operatorname{rel}\left(\boldsymbol{p}_{0}, \mathrm{P}\right), \operatorname{rel}\left(\boldsymbol{p}_{0}, \mathrm{P}\right)\right)\right]
$$

takes into account the energy shift due to forward scattering. This result complies with the quantum linear Boltzmann equation obtained in [12], whose properties were discussed in detail in [4]. 


\section{Immobile tracer particle}

We now consider the opposite situation, corresponding to an infinitely massive test particle, so that the dynamics of the translational degrees of freedom can be neglected. To consider this limit it is convenient to come back to Eq. (5), the expression of the quantum master equation in terms of the complex rate functions $M_{i k}^{j l}\left(\boldsymbol{P}, \boldsymbol{P}^{\prime} ; \boldsymbol{Q}\right)$, which in the limit $M \rightarrow \infty$ when integrated over $\boldsymbol{Q}$ reduce to

$$
\begin{aligned}
M_{i k}^{j l}= & \frac{n_{\mathrm{gas}}}{m^{2}} \chi_{i k}^{j l} \int d \boldsymbol{p} \int d \boldsymbol{p}_{0} \delta\left(\frac{\boldsymbol{p}^{2}-\boldsymbol{p}_{0}^{2}}{2 m}+E_{i}-E_{j}\right) \\
& \times f_{i j}\left(\boldsymbol{p}, \boldsymbol{p}_{0}\right) f_{k l}^{*}\left(\boldsymbol{p}, \boldsymbol{p}_{0}\right),
\end{aligned}
$$

where no dependence on the test particle's momentum is left. The matrix elements of the incoherent part of the quantum Bloch-Boltzmann equation are therefore now given by

$$
\begin{aligned}
& \langle i|\mathcal{L} \rho| k\rangle \\
& =\sum_{j l}\left(\langle j|\rho| l\rangle M_{i k}^{j l}-\frac{1}{2}\langle j|\rho| k\rangle M_{l l}^{j i}-\frac{1}{2}\langle i|\rho| l\rangle M_{j j}^{k l}\right),
\end{aligned}
$$

while the coherent part corresponds to a effective Hamiltonian whose matrix elements in the energy eigenbasis are given by $E_{n}^{i j}=-2 \pi \hbar^{2}\left(n_{\text {gas }} / m_{*}\right) \chi_{i k}^{j k} \int d \boldsymbol{p}_{0} \mu\left(\boldsymbol{p}_{0}\right) \operatorname{Re}\left[f_{i j}\left(\boldsymbol{p}_{0}, \boldsymbol{p}_{0}\right)\right]$, thus confirming the result obtained in [5] for the case of a nondegenerate Hamiltonian.

\section{Quantum classical description}

The limiting expressions of the quantum Bloch-Boltzmann equation, obtained when either the internal or the translational degrees of freedom can be treated as a classical label, correspond to hybrid quantum classical descriptions, which naturally arise when decoherence affects the two kinds of degrees of freedom on different time scales.

When the center-of-mass degrees of freedom can be treated classically, it is convenient to introduce the classical rates

$$
M_{i k}^{j l}(\boldsymbol{P}+\boldsymbol{Q} ; \boldsymbol{Q}):=M_{i k}^{j l}(\boldsymbol{P}+\boldsymbol{Q}, \boldsymbol{P}+\boldsymbol{Q} ; \boldsymbol{Q})
$$

with $M_{i k}^{j l}\left(\boldsymbol{P}, \boldsymbol{P}^{\prime} ; \boldsymbol{Q}\right)$ as in Eq. (6), so that the semiclassical Bloch-Boltzmann equation reads

$$
\begin{aligned}
\frac{d}{d t} \rho(\boldsymbol{P})= & \frac{1}{i \hbar}\left[\sum_{i} \hbar \omega_{i}|i\rangle\langle i|+H_{n}(\boldsymbol{P}), \rho(\boldsymbol{P})\right] \\
& +\sum_{i j k l} \int d \boldsymbol{Q}\left[M_{i k}^{j l}(\boldsymbol{P} ; \boldsymbol{Q}) \mathrm{E}_{i j} \rho(\boldsymbol{P}-\boldsymbol{Q}) \mathrm{E}_{k l}^{\dagger}\right. \\
& \left.-\frac{1}{2} M_{i k}^{j l}(\boldsymbol{P}+\boldsymbol{Q} ; \boldsymbol{Q})\left\{\mathrm{E}_{k l}^{\dagger} \mathrm{E}_{i j}, \rho(\boldsymbol{P})\right\}\right],
\end{aligned}
$$

where $H_{n}(\boldsymbol{P})$ is obtained from Eq. (10) with the replacement $\mathrm{P} \rightarrow \boldsymbol{P}$, and $\rho(\boldsymbol{P})$ denotes a collection of trace class operators in $\mathbb{C}^{n}$ normalized according to $\int d \boldsymbol{P} \operatorname{Tr}_{\mathbb{C}^{n}} \rho(\boldsymbol{P})=1$.

If the classical approximation applies for the internal degrees of freedom, the incoherent term of the master equation giving a quantum description of the translational dynamics only takes the form

$$
\begin{aligned}
\frac{d}{d t} \rho_{i}= & \frac{1}{i \hbar}\left[\frac{\mathrm{P}^{2}}{2 M}+H_{n}^{i}, \rho_{i}\right]+\sum_{j} \int d \boldsymbol{Q} \int_{\boldsymbol{Q}^{\perp}} d \boldsymbol{p} \\
& \times\left[e^{i \boldsymbol{Q} \cdot \mathrm{X} / \hbar} L_{i j}(\boldsymbol{p}, \mathrm{P} ; \boldsymbol{Q}) \rho_{j} L_{i j}(\boldsymbol{p}, \mathrm{P} ; \boldsymbol{Q})^{\dagger} e^{-i \boldsymbol{Q} \cdot \mathrm{X} / \hbar}\right. \\
& \left.-\frac{1}{2}\left\{L_{i j}(\boldsymbol{p}, \mathrm{P} ; \boldsymbol{Q})^{\dagger} L_{i j}(\boldsymbol{p}, \mathrm{P} ; \boldsymbol{Q}), \rho_{i}\right\}\right]
\end{aligned}
$$

Here

$H_{n}^{i}=-2 \pi \hbar^{2} \frac{n_{\text {gas }}}{m_{*}} \int d \boldsymbol{p}_{0} \mu\left(\boldsymbol{p}_{0}\right) \operatorname{Re}\left[f_{i i}\left(\operatorname{rel}\left(\boldsymbol{p}_{0}, \mathrm{P}\right), \operatorname{rel}\left(\boldsymbol{p}_{0}, \mathrm{P}\right)\right)\right]$,

and $\rho_{i}$ denotes a collection of trace class operators in $L^{2}\left(\mathbb{R}^{3}\right)$ normalized according to $\sum_{i=1}^{n} \operatorname{Tr}_{L^{2}\left(\mathbb{R}^{3}\right)} \rho_{i}=1$.

For the case in which all the off-diagonal elements with respect to momentum and internal energy eigenvalues vanish (that is to say, $\left\langle\boldsymbol{P}, i|\rho| \boldsymbol{P}^{\prime}, k\right\rangle=0$ if $\boldsymbol{P} \neq \boldsymbol{P}^{\prime}$ or $i \neq k$ ), the motional state of the test particle is fully characterized by the distribution of the diagonal terms $f_{i}(\boldsymbol{P})=\langle\boldsymbol{P}, i|\rho| \boldsymbol{P}, i\rangle$, which is a classical probability density obeying the classical Markovian master equation

$$
\begin{aligned}
\frac{d}{d t} f_{i}(\boldsymbol{P})= & \sum_{j} \int d \boldsymbol{Q} f_{j}(\boldsymbol{P}-\boldsymbol{Q}) M_{i i}^{j j}(\boldsymbol{P} ; \boldsymbol{Q}) \\
& -f_{i}(\boldsymbol{P}) \sum_{j} \int d \boldsymbol{Q} M_{j j}^{i i}(\boldsymbol{P}+\boldsymbol{Q} ; \boldsymbol{Q}),
\end{aligned}
$$

where the positive quantities $M_{i i}^{j j}(\boldsymbol{P} ; \boldsymbol{Q})$ defined in Eq. (14) can actually be interpreted as the transition rates from an initial momentum $\boldsymbol{P}-\boldsymbol{Q}$ and internal state $j$ to a final momentum $\boldsymbol{P}$ and internal state $i$. This classical Markovian master equation provides the natural generalization of the classical linear Boltzmann equation to a particle with internal degrees of freedom [15].

\section{EFFECT OF INTERNAL DEGREES OF FREEDOM ON CENTER-OF-MASS DECOHERENCE}

The quantum linear Boltzmann equation has proven useful in the description of collisional decoherence, as well as in the evaluation of the index of refraction for matter waves [4,12,16-18]. We now consider the effect of internal degrees of freedom, affecting the collisional interaction between massive test particle and background gas, on the visibility of the interference fringes in a interferometric setup. In particular, we show that the visibility can exhibit oscillations due to non-Markovian effects. The effect of the entanglement between internal and center-of-mass degrees of freedom for the visibility of quantum interference experiments was already considered in [19], in the absence, however, of decoherence effects.

\section{A. Generalized Lindblad structure}

The quantum master equation, Eq. (1), is in Lindblad form: the dynamics of the test particle is Markovian when both translational and internal degrees of freedom are described and 
detected. A different situation emerges if the translational or the internal degrees of freedom, even though they influence the collisional dynamics, are not revealed during the measurement process. In this case, they must be averaged out from the description of the system by means of the partial trace, thus becoming part of the environment. As is well known in the classical case, a non-Markovian dynamical regime becomes Markovian by suitably enlarging the set of degrees of freedom and vice versa. Indeed, a unitary Markovian time evolution for both system and reservoir generally gives a non-Markovian reduced dynamics for the system; the degree of non-Markovianity of the description also depends on where we set the border between system and environment, which ultimately depends on the physical quantities actually measurable by the experimenter. A smaller set of observed degrees of freedom, with respect to those actually involved in the dynamics, can lead from a Markovian to a non-Markovian regime. A general mechanism describing this passage in quantum systems is presented in [6]: a Lindblad structure on a bipartite system can generate a generalized Lindblad structure in the two reduced subsystems, typically describing a non-Markovian dynamics.

In the situation we are considering, the bipartite system is formed by the translational and internal degrees of freedom of the test particle. If the measurements at the output of the detector cannot probe the internal degrees of freedom, the only experimentally accessible quantities are expectations or matrix elements of the statistical operator given by

$$
\varrho(t)=\operatorname{Tr}_{\mathbb{C}^{n}}\{\rho(t)\}=\sum_{i}\langle i|\rho(t)| i\rangle=: \sum_{i} \rho_{i}(t),
$$

where $\rho(t)$ is the statistical operator describing the full dynamics of the test particle. It is easy to see that, if the free
Hamiltonian is nondegenerate, the diagonal matrix elements in the energy basis with respect to the internal degrees of freedom of the master equation lead to Eq. (16), that is, a coupled system of equations for the collection $\left\{\rho_{i}(t)\right\}_{i}$ of trace class operators on $L^{2}\left(\mathbb{R}^{3}\right)$. This system of equations has a generalized Lindblad structure [9] and, therefore, it can also describe highly non-Markovian dynamics for the statistical operator $\varrho(t)$ given by Eq. (19). Indeed, there is generally no closed evolution equation for $\varrho(t)$, but from the knowledge of the initial collection $\left\{\rho_{i}(0)\right\}_{i}$ the generalized Lindblad structure allows the collection $\left\{\rho_{i}(t)\right\}_{i}$ to be obtained at time $t$; therefore, through Eq. (19), $\varrho(t)$ is also obtained. In the next paragraph, we explicitly point out non-Markovian behavior described by the generalized Lindblad structure, which express the effect of correlations between internal and translational degrees of freedom on the visibility of interference fringes for superpositions of motional states. A complementary situation was considered in [20], where the effect of collisional decoherence on internal-state superpositions of a cold gas was studied in detail.

In typical interferometric experiments, the test particle is much more massive than the particles of the background gas. The dependence on the momentum operator in the Lindblad operators describing the collisional dynamics and in the Hamiltonian part determining the energy shift can therefore be replaced by a fixed value $\boldsymbol{P}_{0}$, which represents the initial momentum of the test particle entering the interferometer. Taking the diagonal matrix elements of the general form of the master equation given by Eq. (1) and specified by Eqs. (10) and (9) and assuming nondegeneracy of the internal energy eigenvalues, one finally obtains for the collection of operators $\rho_{i}(t)=\langle i|\rho(t)| i\rangle$ the following coupled system of equations:

$$
\frac{d}{d t} \rho_{i}(t)=\frac{1}{i \hbar}\left[\frac{\mathrm{P}^{2}}{2 M}, \rho_{i}(t)\right]+\sum_{j}\left(\Gamma_{\boldsymbol{P}_{0}}^{i j} \int d \boldsymbol{Q} \mathcal{P}_{\boldsymbol{P}_{0}}^{i j}(\boldsymbol{Q}) \mathrm{e}^{i \boldsymbol{Q} \cdot \mathrm{X} / \hbar} \rho_{j}(t) \mathrm{e}^{-i \boldsymbol{Q} \cdot \mathrm{X} / \hbar}-\Gamma_{\boldsymbol{P}_{0}}^{j i} \rho_{i}(t)\right),
$$

where $\mathcal{P}_{\boldsymbol{P}}^{i j}$ and $\Gamma_{\boldsymbol{P}}^{j i}$ are probability densities and transition rates defined by

$$
\mathcal{P}_{\boldsymbol{P}}^{i j}(\boldsymbol{Q}):=\frac{M_{i i}^{j j}(\boldsymbol{P} ; \boldsymbol{Q})}{\int d \boldsymbol{Q} M_{i i}^{j j}(\boldsymbol{P}+\boldsymbol{Q} ; \boldsymbol{Q})}
$$

and

$$
\Gamma_{\boldsymbol{P}}^{i j}:=\int d \boldsymbol{Q} M_{i i}^{j j}(\boldsymbol{P}+\boldsymbol{Q} ; \boldsymbol{Q}),
$$

respectively. Note that, at variance with Eq. (16), we are now not assuming a classical dynamics for the internal degrees of freedom; instead we focus on the diagonal matrix elements of the internal states only since the latter are enough to determine the non-Markovian dynamics of the motional state according to Eq. (19). The fact that the positive quantities $M_{i i}^{j j}(\boldsymbol{P} ; \boldsymbol{Q})$ are transition rates implies that $\mathcal{P}_{\boldsymbol{P}}^{i j}(\boldsymbol{Q})$ can be interpreted as the probability distribution function for a test particle with momentum $\boldsymbol{P}$ and internal energy eigenstate $j$ to exchange a momentum $\boldsymbol{Q}$, and to go into the internal state $i$ due to a collision with the gas. On the same footing, $\Gamma_{\boldsymbol{P}}^{i j}$ can be interpreted as the total transition rate for a test particle with momentum $\boldsymbol{P}$ and internal state $j$ to go to a fixed final internal energy eigenstate $i$.

\section{B. Explicit solutions in position representation}

We now describe the visibility reduction predicted by the generalized Lindblad structure in Eq. (20) obtained from the quantum Bloch-Boltzmann equation, Eq. (1), in the limit of a very massive test particle. To obtain the formula describing the fringe visibility in an explicit way, we need to solve the equation of motion in the position representation. Starting from Eq. (20) and omitting for simplicity the explicit dependence on the classical label $\boldsymbol{P}_{0}$ denoting the momentum of the test 
particle, we obtain

$$
\begin{aligned}
\frac{d}{d t} \rho_{i}\left(\boldsymbol{X}, \boldsymbol{X}^{\prime}, t\right)= & \frac{1}{i \hbar}\left(\Delta_{\boldsymbol{X}}-\Delta_{\boldsymbol{X}^{\prime}}\right) \rho_{i}\left(\boldsymbol{X}, \boldsymbol{X}^{\prime}, t\right) \\
& +\sum_{j}\left(\Gamma^{i j} \Phi^{i j}\left(\boldsymbol{X}-\boldsymbol{X}^{\prime}\right) \rho_{j}\left(\boldsymbol{X}, \boldsymbol{X}^{\prime}, t\right)\right. \\
& \left.-\Gamma^{j i} \rho_{i}\left(\boldsymbol{X}, \boldsymbol{X}^{\prime}, t\right)\right)
\end{aligned}
$$

where $\rho_{i}\left(\boldsymbol{X}, \boldsymbol{X}^{\prime}, t\right)$ denotes the matrix element $\left\langle\boldsymbol{X}\left|\rho_{i}(t)\right| \boldsymbol{X}^{\prime}\right\rangle$ and $\Phi^{i j}\left(\boldsymbol{X}-\boldsymbol{X}^{\prime}\right)$ is the characteristic function of the probability density $\mathcal{P}^{i j}(\boldsymbol{Q})$ [21], that is, its Fourier transform

$$
\Phi^{i j}\left(\boldsymbol{X}-\boldsymbol{X}^{\prime}\right)=\int d \boldsymbol{Q} e^{i\left(\boldsymbol{X}-\boldsymbol{X}^{\prime}\right) \cdot \boldsymbol{Q} / \hbar} \mathcal{P}^{i j}(\boldsymbol{Q}) .
$$

We now consider a few cases in which Eq. (23) can be solved analytically, so as to obtain an exact expression for the visibility and to show different possible qualitative behavior.

\section{N-level system}

When the collisions are purely elastic, so that they do not lead to transitions between different internal states, the scattering rates satisfy $\Gamma^{i j}=\delta_{i j} \Gamma^{i i}$. This is the case when the energy exchanges involved in the single collisions are much smaller than the typical separation of the internal energy levels [13]. The equations for the different $\rho_{i}$ then become uncoupled and take the following form:

$$
\begin{aligned}
\frac{d}{d t} \rho_{i}\left(\boldsymbol{X}, \boldsymbol{X}^{\prime}, t\right)= & \frac{1}{i \hbar}\left(\Delta_{\boldsymbol{X}}-\Delta_{\boldsymbol{X}^{\prime}}\right) \rho_{i}\left(\boldsymbol{X}, \boldsymbol{X}^{\prime}, t\right) \\
& -\Gamma^{i i}\left(1-\Phi^{i i}\left(\boldsymbol{X}-\boldsymbol{X}^{\prime}\right)\right) \rho_{i}\left(\boldsymbol{X}, \boldsymbol{X}^{\prime}, t\right) .
\end{aligned}
$$

The preceding equation can be conveniently solved by introducing the function [22]

$$
\chi_{i}(\boldsymbol{\lambda}, \boldsymbol{\mu}, t):=\operatorname{Tr}\left\{\rho_{i}(t) e^{i(\lambda \cdot \mathrm{X}+\boldsymbol{\mu} \cdot \mathrm{P}) / \hbar}\right\},
$$

where $\mathrm{X}$ and $\mathrm{P}$ as usual denote position and momentum operators of the test particle. In such a way, Eq. (25) leads to

$$
\partial_{t} \chi_{i}(\lambda, \mu, t)=\left[\frac{\lambda}{M} \cdot \partial_{\mu}-\Gamma^{i i}\left(1-\Phi^{i i}(\boldsymbol{\mu})\right)\right] \chi_{i}(\boldsymbol{\lambda}, \boldsymbol{\mu}, t),
$$

which is an equation of first order solved by

$$
\chi_{i}(\boldsymbol{\lambda}, \boldsymbol{\mu}, t)=\chi_{i}^{0}(\lambda, \lambda t / M+\mu) e^{-\Gamma^{i i} \int_{0}^{t}\left(1-\Phi^{i i}\left(\lambda\left(t-t^{\prime}\right) / M+\mu\right)\right) d t^{\prime}},
$$

where the function $\chi_{i}^{0}(\lambda, \lambda t / M+\mu)$ obeys the free equation $\partial_{t} \chi_{i}(\boldsymbol{\lambda}, \boldsymbol{\mu}, t)=(\boldsymbol{\lambda} / M) \cdot \partial_{\boldsymbol{\mu}} \chi_{i}(\boldsymbol{\lambda}, \boldsymbol{\mu}, t)$. Inverting Eq. (26) by taking the Fourier transform with respect to $\lambda$,

$\rho_{i}\left(\boldsymbol{X}, \boldsymbol{X}^{\prime}, t\right)=\int \frac{d \boldsymbol{\lambda}}{(2 \pi \hbar)^{3}} e^{-i \boldsymbol{\lambda} \cdot\left(\boldsymbol{X}+\boldsymbol{X}^{\prime}\right) / 2 \hbar} \chi_{i}\left(t, \boldsymbol{\lambda}, \boldsymbol{X}-\boldsymbol{X}^{\prime}\right)$,

we obtain the exact solution

$$
\begin{aligned}
\rho_{i}\left(\boldsymbol{X}, \boldsymbol{X}^{\prime}, t\right)= & \int \frac{d \boldsymbol{s} d \boldsymbol{\lambda}}{(2 \pi \hbar)^{3}} e^{-i \boldsymbol{\lambda} \cdot \boldsymbol{s} / \hbar} e^{-\Gamma^{i i} \int_{0}^{t}\left(1-\Phi^{i i}\left(\lambda\left(t-t^{\prime}\right) / M+\boldsymbol{X}-\boldsymbol{X}^{\prime}\right)\right) d t^{\prime}} \\
& \times \rho_{i}^{0}\left(\boldsymbol{X}+\boldsymbol{s}, \boldsymbol{X}^{\prime}+\boldsymbol{s}, t\right)
\end{aligned}
$$

expressed in terms of an integral of the freely evolved subcollections $\rho_{i}^{0}\left(\boldsymbol{X}, \boldsymbol{X}^{\prime}, t\right)$ with a suitable kernel, where we have set

$$
\begin{aligned}
& \rho_{i}^{0}\left(\boldsymbol{X}, \boldsymbol{X}^{\prime}, t\right) \\
& \quad=\int \frac{d \lambda}{(2 \pi \hbar)^{3}} e^{-i \lambda \cdot\left(\boldsymbol{X}+\boldsymbol{X}^{\prime}\right) / 2 \hbar} \chi^{0}\left(\boldsymbol{\lambda}, \boldsymbol{X}-\boldsymbol{X}^{\prime}, t\right) \\
& \quad=\left\langle\boldsymbol{X}\left|\exp \left(-\frac{i}{\hbar} \frac{\mathrm{P}^{2}}{2 M} t\right) \rho_{i}(0) \exp \left(+\frac{i}{\hbar} \frac{\mathrm{P}^{2}}{2 M} t\right)\right| \boldsymbol{X}^{\prime}\right\rangle
\end{aligned}
$$

and $\rho_{i}(0)=\langle i|\rho(0)| i\rangle$. The evolution of the statistical operator given by Eq. (19) is obtained by summing the different $\rho_{i}\left(\boldsymbol{X}, \boldsymbol{X}^{\prime}, t\right)$ over the discrete index $i$. For an initial state given by a product state between the translational and the internal part, so that $\rho_{i}(0)=p_{i} \varrho(0)$, we finally obtain

$$
\begin{aligned}
& \varrho\left(\boldsymbol{X}, \boldsymbol{X}^{\prime}, t\right) \\
& =\sum_{i} p_{i} \int \frac{d \boldsymbol{s} d \boldsymbol{\lambda}}{(2 \pi \hbar)^{3}} e^{-i \lambda \cdot \boldsymbol{s} / \hbar} e^{-\Gamma^{i i} \int_{0}^{t}\left(1-\Phi^{i i}\left(\lambda\left(t-t^{\prime}\right) / M+\boldsymbol{X}-\boldsymbol{X}^{\prime}\right)\right) d t^{\prime}} \\
& \quad \times \varrho^{0}\left(\boldsymbol{X}+\boldsymbol{s}, \boldsymbol{X}^{\prime}+\boldsymbol{s}, t\right) .
\end{aligned}
$$

This result reduces to the standard Markovian situation, when either only one value of $p_{i}$ is different from zero (and therefore equal to 1), or the rates are all equal. This limiting cases describes situations in which the initial state is in a specific internal state or the collisions do not depend on the internal state of the tracer particle.

\section{Two-level system}

For the case of a two-level system, a natural situation corresponds to inelastic scattering taking place only when the test particle gets de-excited, so that only one of the two scattering rates is different from zero. This case can still be treated analytically. Assuming $\Gamma^{21}=0$, the equation for $\chi_{2}(t, \lambda, \mu)$ gets closed and is solved by

$$
\begin{aligned}
\chi_{2}(\boldsymbol{\lambda}, \boldsymbol{\mu}, t)= & \chi_{2}^{0}(\boldsymbol{\lambda}, \boldsymbol{\lambda} t / M+\boldsymbol{\mu}) e^{-\Gamma^{12} t} \\
& \times e^{-\Gamma^{22} \int_{0}^{t}\left(1-\Phi^{22}\left(\lambda\left(t-t^{\prime}\right) / M+\mu\right)\right) d t^{\prime}} .
\end{aligned}
$$

The equation for $\chi_{1}(\boldsymbol{\lambda}, \boldsymbol{\mu}, t)$ then reads

$$
\begin{aligned}
\partial_{t} \chi_{1}(\lambda, \mu, t)= & {\left[\frac{\lambda}{M} \cdot \partial_{\mu}-\Gamma^{11}\left(1-\Phi^{11}(\boldsymbol{\mu})\right)\right] \chi_{1}(\boldsymbol{\lambda}, \boldsymbol{\mu}, t) } \\
& +\Gamma^{12} \Phi^{12}(\boldsymbol{\mu}) \chi_{2}(\boldsymbol{\lambda}, \boldsymbol{\mu}, t)
\end{aligned}
$$

and its solution is given by

$$
\begin{aligned}
\chi_{1}(\boldsymbol{\lambda}, \boldsymbol{\mu}, t) \\
=e^{-\Gamma^{11} \int_{0}^{t}\left(1-\Phi^{11}\left(\lambda\left(t-t^{\prime}\right) / M+\mu\right)\right) d t^{\prime}}\left\{\chi_{1}^{0}(\lambda, \lambda t / M+\mu)\right. \\
\quad+\Gamma^{12} \int_{0}^{t}\left[e^{\Gamma^{11} \int_{0}^{t^{\prime}}\left(1-\Phi^{11}\left(\lambda\left(t-t^{\prime \prime}\right) / M+\mu\right)\right) d t^{\prime \prime}}\right. \\
\left.\left.\quad \times \Phi^{12}\left(\lambda\left(t-t^{\prime}\right) / M+\mu\right) \chi_{2}\left(t^{\prime}, \lambda, \lambda\left(t-t^{\prime}\right) / M+\mu\right)\right] d t^{\prime}\right\} .
\end{aligned}
$$

This formula explicitly shows that $\chi_{1}(\boldsymbol{\lambda}, \boldsymbol{\mu}, t)$ depends on the function $\chi_{2}(\boldsymbol{\lambda}, \boldsymbol{\mu}, \cdot)$ evaluated over the whole time interval between 0 and $t$, a typical signature of non-Markovian dynamics. Assuming once again that the initial state is characterized by $\rho_{i}(0)=p_{i} \varrho(0)$, the statistical operator describing the translational degrees of freedom of the test particle is given 
at time $t$ by the expression

$$
\begin{aligned}
\varrho\left(\boldsymbol{X}, \boldsymbol{X}^{\prime}, t\right)= & \int \frac{d \boldsymbol{s} d \boldsymbol{\lambda}}{(2 \pi \hbar)^{3}} e^{-i \lambda \cdot s / \hbar} \varrho^{0}\left(\boldsymbol{X}+\boldsymbol{s}, \boldsymbol{X}^{\prime}+s, t\right) \\
& \times\left\{p_{2} e^{-\Gamma^{12} t} e^{-\Gamma^{22} \int_{0}^{t}\left(1-\Phi^{22}\left(\lambda\left(t-t^{\prime}\right) / M+\boldsymbol{X}-\boldsymbol{X}^{\prime}\right)\right) d t^{\prime}}\right. \\
& +p_{1} e^{-\Gamma^{11} \int_{0}^{t}\left(1-\Phi^{11}\left(\lambda\left(t-t^{\prime}\right) / M+\boldsymbol{X}-\boldsymbol{X}^{\prime}\right)\right) d t^{\prime}} \\
& +p_{2} \Gamma^{12} e^{-\Gamma^{11} \int_{0}^{t}\left(1-\Phi^{11}\left(\lambda\left(t-t^{\prime}\right) / M+\boldsymbol{X}-\boldsymbol{X}^{\prime}\right)\right) d t^{\prime}} \\
& \times \int_{0}^{t}\left(e^{-\Gamma^{12} t^{\prime}} e^{-\Gamma^{22} \int_{0}^{t^{\prime}}\left(1-\Phi^{22}\left(\lambda\left(t-t^{\prime \prime}\right) / M+\boldsymbol{X}-\boldsymbol{X}^{\prime}\right)\right) d t^{\prime \prime}}\right. \\
& \times e^{\Gamma^{11} \int_{0}^{t^{\prime}}\left(1-\Phi^{11}\left(\lambda\left(t-t^{\prime \prime}\right) / M+\boldsymbol{X}-\boldsymbol{X}^{\prime}\right)\right) d t^{\prime \prime}} \\
& \left.\left.\times \Phi^{12}\left(\lambda\left(t-t^{\prime}\right) / M+\boldsymbol{X}-\boldsymbol{X}^{\prime}\right)\right) d t^{\prime}\right\}
\end{aligned}
$$

\section{Nonexponential visibility reduction}

We can now explicitly present the visibility reduction predicted by the generalized Lindblad structure obtained from the quantum master equation for a test particle with internal degrees of freedom. Our aim is to obtain an exact expression for the loss of visibility in a double-slit arrangement as a function of the time of interaction with the environment, and to illustrate by means of example how the presence of the various scattering channels, corresponding to the different internal states, can actually lead to non-Markovian behaviors. In particular we consider the situation of purely elastic collisions in full generality, also allowing for inelastic scattering in the case of a two-level system. While the experimental setting is always taken to be the same, the different number of internal degrees of freedom involved and the presence or absence of inelastic scattering events lead to more or less marked nonexponential behaviors in the reduction of the visibility fringes.

\section{Visibility formula}

We first derive a formula for the visibility reduction in the case of a double-slit experiment in the far-field approximation. A beam of particles moves toward a grating perpendicular to its direction of propagation, and with two identical slits separated by a distance $d$, finally reaching a detector where the fringes of interference are observed. During the flight through the interferometer, the beam particles interact through collisions with the environment in the background, thus undergoing decoherence. We consider an initial product state, so that in the notation of Eq. (20) one has $\rho_{i}(0)=p_{i} \varrho(0)$, where $\varrho(0)$ describes the translational degrees of freedom. If after the passage through the collimation slits the test particle is described by $\rho_{\mathrm{sl}}$, then the double-slit grating prepares the initial state [23]

$$
\varrho(0)=2 \cos \left(\frac{\mathrm{P} \cdot \boldsymbol{d}}{2 \hbar}\right) \rho_{\mathrm{sl}} \cos \left(\frac{\mathrm{P} \cdot \boldsymbol{d}}{2 \hbar}\right) .
$$

Setting

$$
\varrho(\boldsymbol{X}, \boldsymbol{X}, t)=\langle\boldsymbol{X}|\mathcal{U}(t)[\varrho(0)]| \boldsymbol{X}\rangle:=I(\boldsymbol{X}),
$$

we consider the quantity

$$
\mathcal{V}=\frac{I_{\max }-I_{\min }}{I_{\max }+I_{\min }}
$$

which describes the reduction of the interference pattern with respect to the free case. Exploiting the fact that the time evolution generated by Eq. (31) is covariant under translations $[4,24]$, so that

$$
\mathcal{U}(t)\left[e^{i \mathrm{P} \cdot \boldsymbol{a} / \hbar} \varrho e^{-i \mathrm{P} \cdot \boldsymbol{a} / \hbar}\right]=e^{i \mathrm{P} \cdot \boldsymbol{a} / \hbar} \mathcal{U}(t)[\varrho] e^{-i \mathrm{P} \cdot \boldsymbol{a} / \hbar},
$$

one has, using Eq. (36),

$$
\mathcal{V}=\frac{2\left|\left\langle\boldsymbol{X}-\frac{1}{2} \boldsymbol{d}\left|\mathcal{U}(t)\left[\rho_{\mathrm{sl}} e^{-i \mathrm{P} \cdot \boldsymbol{d} / \hbar}\right]\right| \boldsymbol{X}-\frac{1}{2} \boldsymbol{d}\right\rangle\right|}{\left\langle\boldsymbol{X}-\frac{1}{2} \boldsymbol{d}\left|\mathcal{U}(t)\left[\rho_{\mathrm{sl}}\right]\right| \boldsymbol{X}-\frac{1}{2} \boldsymbol{d}\right\rangle+\left\langle\boldsymbol{X}+\frac{1}{2} \boldsymbol{d}\left|\mathcal{U}(t)\left[\rho_{\mathrm{sl}}\right]\right| \boldsymbol{X}+\frac{1}{2} \boldsymbol{d}\right\rangle},
$$

where $t$ is now the time employed by the test particle to reach the detector. Indeed this result remains true for any translationcovariant time evolution.

For an initial factorized state of the test particle, we can exploit Eq. (31) to obtain a closed formula for the time evolution operator $\mathcal{U}(t)$ depending on the initial internal state, that is, on the coefficients $p_{i}$ appearing in $\rho_{i}(0)=p_{i} \varrho(0)$ : the numerator of Eq. (38) then reads

$$
\begin{gathered}
2 \mid \sum_{i} p_{i} \int \frac{d \boldsymbol{s} d \boldsymbol{\lambda}}{(2 \pi \hbar)^{3}} e^{-i \boldsymbol{\lambda} \cdot \boldsymbol{s} / \hbar}\left\langle\boldsymbol{X}-\frac{\boldsymbol{d}}{2}+\boldsymbol{s}\right| \mathcal{U}_{0}(t)\left[\rho_{\mathrm{sl}} e^{-i \mathrm{P} \cdot \boldsymbol{d} / \hbar}\right] \\
\times\left|\boldsymbol{X}-\frac{\boldsymbol{d}}{2}+\boldsymbol{s}\right\rangle e^{-\Gamma^{i i} \int_{0}^{t}\left(1-\Phi^{i i}\left(\boldsymbol{\lambda}\left(t-t^{\prime}\right) / M\right)\right) d t^{\prime}} \mid
\end{gathered}
$$

where $\mathcal{U}_{0}(t)$ is the free evolution operator of the translational degrees of freedom, so that

$$
\begin{gathered}
\left\langle\boldsymbol{X}-\frac{\boldsymbol{d}}{2}+\boldsymbol{s}\left|\mathcal{U}_{0}(t)\left[\rho_{\mathrm{s} 1} e^{-i \mathrm{P} \cdot \boldsymbol{d} / \hbar}\right]\right| \boldsymbol{X}-\frac{\boldsymbol{d}}{2}+\boldsymbol{s}\right\rangle \\
=\left\langle\boldsymbol{X}-\frac{\boldsymbol{d}}{2}+\boldsymbol{s}\left|\mathcal{U}_{0}(t)\left[\rho_{\mathrm{s} l}\right]\right| \boldsymbol{X}+\frac{\boldsymbol{d}}{2}+\boldsymbol{s}\right\rangle .
\end{gathered}
$$

The latter expression can also be written

$$
\begin{aligned}
\langle\boldsymbol{X} & \left.-\frac{\boldsymbol{d}}{2}\left|\mathcal{U}_{0}(t)\left[\rho_{\mathrm{sl}}\right]\right| \boldsymbol{X}+\frac{\boldsymbol{d}}{2}\right\rangle \\
= & \left(\frac{M}{t}\right)^{3} e^{-i M \boldsymbol{d} \cdot \boldsymbol{X} /(\hbar t)} \int \frac{d \boldsymbol{Y} d \boldsymbol{Y}^{\prime}}{(2 \pi \hbar)^{3}} e^{i M\left(\boldsymbol{Y}^{2}-\boldsymbol{Y}^{\prime 2}\right) /(2 \hbar t)} \\
& \times e^{-i M \boldsymbol{X} \cdot\left(\boldsymbol{Y}-\boldsymbol{Y}^{\prime}\right) /(\hbar t)} e^{i M \boldsymbol{d} \cdot\left(\boldsymbol{Y}+\boldsymbol{Y}^{\prime}\right) /(2 \hbar t)}\left\langle\boldsymbol{Y}\left|\rho_{\mathrm{sl}}\right| \boldsymbol{Y}^{\prime}\right\rangle
\end{aligned}
$$

assuming, due to symmetry, $\operatorname{Tr}\left(\mathrm{X} \rho_{\mathrm{sl}}\right)=0$. 
This formula enables us to implement the far-field approximation. In fact, let $\sigma$ be the width of the two slits, so that the integrand is negligible if $\boldsymbol{Y}$ takes values outside the support of $\rho_{\mathrm{sl}}$ (and similarly for $\boldsymbol{Y}^{\prime}$ ), then $M \boldsymbol{Y}^{2} /(\hbar t) \lesssim M \sigma^{2} /(\hbar t)$ and therefore, for a time long enough such that $\hbar t / M \gg \sigma^{2}$, the first exponential can be disregarded. The same applies for the last exponential if $\hbar t / M \gg \sigma d$. For times longer than $\max \left\{M \sigma^{2} / \hbar, M \sigma d / \hbar\right\}$, corresponding to the far-field approximation, we get******

$$
\begin{gathered}
\left\langle\boldsymbol{X}-\frac{\boldsymbol{d}}{2}\left|\mathcal{U}_{0}(t)\left[\rho_{\mathrm{sl}}\right]\right| \boldsymbol{X}+\frac{\boldsymbol{d}}{2}\right\rangle \\
\approx\left(\frac{M}{t}\right)^{3} e^{-i M \boldsymbol{d} \cdot \boldsymbol{X} /(\hbar t)} \tilde{\rho}_{\mathrm{sl}}\left(\frac{M}{t} \boldsymbol{X}\right),
\end{gathered}
$$

where $\tilde{\rho}_{\mathrm{sl}}(\cdot)$ is the distribution function for the momentum of the particle in the state $\rho_{\mathrm{sl}}$,

$$
\tilde{\rho}_{\mathrm{sl}}\left(\frac{M}{t} \boldsymbol{X}\right)=\int \frac{d \boldsymbol{Y} d \boldsymbol{Y}^{\prime}}{(2 \pi \hbar)^{3}} e^{-i M \boldsymbol{X} \cdot\left(\boldsymbol{Y}-\boldsymbol{Y}^{\prime}\right) /(\hbar t)}\left\langle\boldsymbol{Y}\left|\rho_{\mathrm{sl}}\right| \boldsymbol{Y}^{\prime}\right\rangle .
$$

The equivalence between the assumption $\hbar t / M \gg \sigma^{2}$ and the far-field approximation $L \gg \sigma^{2} / \lambda$, where $\lambda=\hbar / P_{z}$ is the wavelength associated with the test particle and $L$ is the distance between grating and detector, is easily seen from the relation $L=p_{z} t / M$, where $p_{z}$ is the component along the $z$ direction of the massive particle, assumed to be constant. Substituting Eq. (39) in the numerator of Eq. (38) and using the approximation $\tilde{\rho}_{\mathrm{sl}}(M(X+s) / t) \approx \tilde{\rho}_{\mathrm{sl}}(M X / t)$ which is valid because of the localization of the state $\rho_{\text {sl }}$, we can easily perform the integrals over $s$ and $\lambda$, thus finally obtaining

$$
2\left(\frac{M}{t}\right)^{3}\left|\tilde{\rho}_{\mathrm{sl}}\left(\frac{M}{t} \boldsymbol{X}\right)\right|\left|\sum_{i=1}^{n} p_{i} e^{-\Gamma^{i i} \int_{0}^{t}\left(1-\Phi^{i i}\left(\boldsymbol{d}^{\frac{t^{\prime}-t}{t}}\right)\right) d t^{\prime}}\right| .
$$

For the denominator of Eq. (38), one can proceed in an analogous way, using

$$
\begin{gathered}
\left\langle\boldsymbol{X} \pm \frac{1}{2} \boldsymbol{d}+\boldsymbol{s}\left|\mathcal{U}_{0}(t)\left[\rho_{\mathrm{sl}}\right]\right| \boldsymbol{X} \pm \frac{1}{2} \boldsymbol{d}+\boldsymbol{s}\right\rangle \\
\approx\left(\frac{M}{t}\right)^{3} \tilde{\rho}_{\mathrm{sl}}\left(\frac{M}{t} \boldsymbol{X}\right)
\end{gathered}
$$

and performing the integral over $\lambda$, further observing that $\Phi^{i j}(0)=1$ for the normalization of $\mathcal{P}^{i j}(\boldsymbol{Q})$.

\section{Nonexponential behaviors}

The desired expression for the visibility in the absence of inelastic scattering and for an arbitrary number of channels, $n$, thus reads

$$
\mathcal{V}=\left|\sum_{i=1}^{n} p_{i} e^{-\Gamma^{i i} \int_{0}^{t}\left(1-\Phi^{i i}\left(\boldsymbol{d}^{t^{\prime}-t} t\right)\right)} d t^{\prime}\right|,
$$

where we recall that the probabilities $p_{i}$ give the weight of the different internal states in the initial preparation. The dependence on $t$ in this formula can be easily made explicit with the change of variable $t^{\prime} / t=s$ so that one has

$$
\mathcal{V}=\left|\sum_{i=1}^{n} p_{i} e^{-\Gamma^{i i}\left(1-\int_{0}^{1} \Phi^{i i}(d(s-1)) d s\right) t}\right|
$$

From Eq. (43) one can easily see the difference between the Markovian situation, corresponding to $n=1$, and the general case. If there is just one term in the sum, the modulus simply picks out the real part of the characteristic function in the exponential and Eq. (43) describes an exponential decay in time with a rate $\Gamma\left(1-\int_{0}^{1} \operatorname{Re}\{\Phi[\boldsymbol{d}(s-1)]\} d s\right)$. This can happen if only one internal energy state is populated in the initial preparation or the scattering events are actually independent of the internal state. If there are at least two terms, the modulus can generate oscillating terms as a consequence of the interference of the different phases arising since the functions $\Phi^{i i}$ are generally complex valued. Even if the imaginary parts of the characteristic functions are zero (i.e., the distribution functions of the exchanged momenta are even), Eq. (43) can describe highly nonexponential behavior. In this case in fact it reduces to

$$
\mathcal{V}=\sum_{i=1}^{n} p_{i} e^{-\Gamma^{i i}\left(1-\int_{0}^{1} \Phi^{i i}(d(s-1)) d s\right) t}
$$

that is, the sum of different exponential functions. As shown in $[6,25]$, this kind of relations can describe behavior very different from the exponential one.

Let us consider in more detail the case of a two-level system. Introducing the notation

$$
\begin{aligned}
& \alpha^{i}:=\operatorname{Re} \int_{0}^{1}\left\{\Phi^{i i}[\boldsymbol{d}(s-1)]\right\} d s \\
& \beta^{i}:=\operatorname{Im} \int_{0}^{1}\left\{\Phi^{i i}[\boldsymbol{d}(s-1)]\right\} d s,
\end{aligned}
$$

the visibility reduction is explicitly given by

$$
\begin{aligned}
\mathcal{V}= & \left\{p_{1}^{2} e^{-2 \Gamma^{11}\left(1-\alpha^{1}\right) t}+p_{2}^{2} e^{-2 \Gamma^{22}\left(1-\alpha^{2}\right) t}+2 p_{1} p_{2} e^{-\Gamma^{11}\left(1-\alpha^{1}\right) t}\right. \\
& \left.\times e^{-\Gamma^{22}\left(1-\alpha^{2}\right) t} \cos \left[\left(\Gamma^{11} \beta^{1}-\Gamma^{22} \beta^{2}\right) t\right]\right\}^{1 / 2}
\end{aligned}
$$

This formula describes a decrease modulated by the oscillations produced by the cosine function. To illustrate this behavior, in Fig. 1(a) we plot the visibility as a function of time, considering by means of example two Gaussian distributions. Note that the appearance of the oscillations depends on a nonvanishing mean value for the distribution functions $\mathcal{P}^{i i}(\boldsymbol{Q})$ given by Eq. (21), which describe the state-dependent momentum transfers. This feature corresponds to a preferred direction in the net momentum transfer between test particle and environment, as happens, for example, by the interaction with a laser beam [26], where the asymmetry in the single interaction channel is determined in this case by the direction of propagation.

The behavior described by Eq. (43) for an $n$-level system is illustrated in Fig. 1(b), where we show how the increased number of levels can strongly suppress the oscillations and lead to a reduction of the visibility. The dashed lines represent the exponential decays pertaining to the Markovian situation arising if only one of the internal energy states is initially populated, where the one with the highest or lowest decoherence rate corresponds to the lower or upper dashed line, respectively. It appears that with growing $n$ the interference between the contributions of the different channels to Eq. (43) rapidly determines a decay of the visibility sensibly faster than that occurring for the corresponding Markovian single-channel dynamics. Indeed, Figs. 1(a) and 1(b) correspond to the same 

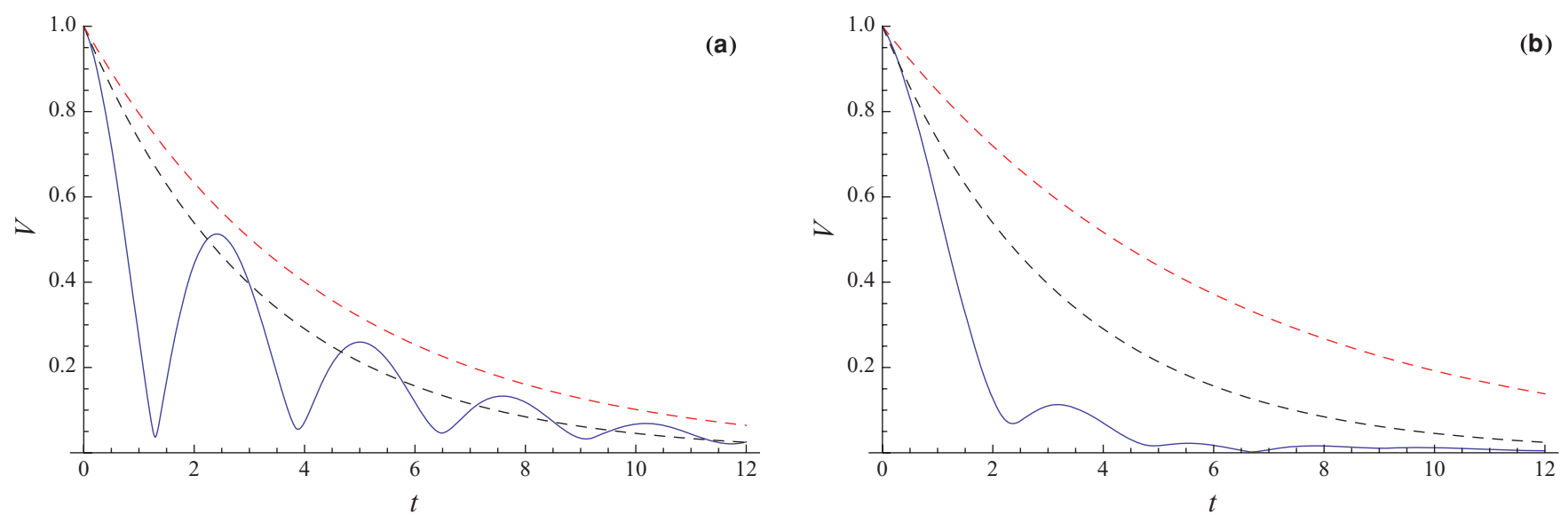

FIG. 1. (Color online) Plot of the visibility in a double-slit arrangement as a function of the interaction time with the environment, in arbitrary units, for the case of elastic scattering events only, according to Eq. (43) and with number of channels increasing from left to right. The dashed lines represent the Markovian exponential decays occurring if a single elastic channel prevails on the others, the ones with the highest and lowest decay rate corresponding to lower and upper line, respectively. (a) Visibility for $n=2$ elastic channels, according to Eq. (46). It appears as a nonmonotonic decay as a consequence of the interference between the contributions of the two different elastic channels. The coefficients $\alpha^{i}$ and $\beta^{i}$ defined in Eq. (45) are calculated for two Gaussian distributions $\mathcal{P}^{11}(\boldsymbol{Q})$ and $\mathcal{P}^{22}(\boldsymbol{Q})$ of the exchanged momenta. Taking $\boldsymbol{d}=d \hat{z}$ as the direction of propagation inside the interferometer, we only need to specify the mean and the variance of the exchanged momenta along this axis, $\mu_{i i}$ and $\sigma_{i i}(i=1,2)$, respectively. The plot is for $p_{1}=p_{2}=\frac{1}{2}$, while $\Gamma^{11}=\Gamma^{22}=10, d=1, \sigma_{11}=\sigma_{22}=0.1, \mu_{11}=-0.2$, and $\mu_{22}=0.3$ in a.u.. (b) Visibility for $n=8$ elastic channels according to the general expression of Eq. (43). The characteristic functions $\Phi^{i i}$ are calculated starting from Gaussian distributions, assuming equal rates $\Gamma^{i i}=10$ and equal variances $\sigma_{i i}=0.1$ in arbitrary units as in (a). The $p_{i}$ are uniformly distributed and the means $\mu_{i i}$ are equally spaced in the range from -0.2 to 0.3 in a.u..

interaction strength but differ in the number of involved degrees of freedom, ranging from $n=2$ to $n=8$.

Relying on the results of Sec. III B 2, one can also obtain an expression of the visibility in the presence of inelastic scattering for a two-level system. Indeed starting from Eq. (35) and following the same procedure as before, one obtains

$$
\begin{aligned}
\mathcal{V}= & \mid e^{-\Gamma^{12} t}+\Gamma^{12} e^{-\Gamma^{11} t \int_{0}^{1}\left(1-\Phi^{11}(\boldsymbol{d}(s-1)) d s\right.} \\
& \times \int_{0}^{t}\left(e^{-\Gamma^{12} t^{\prime}} e^{+\Gamma^{11} \int_{0}^{t^{\prime}}\left(1-\Phi^{11}\left(\boldsymbol{d}\left(t^{\prime \prime}-t\right) / t\right)\right) d t^{\prime \prime}}\right. \\
& \left.\times \Phi^{12}\left(\boldsymbol{d} \frac{t^{\prime}-t}{t}\right)\right) d t^{\prime} \mid
\end{aligned}
$$

where for simplicity $p_{2}=1$, and we have taken $\Gamma^{22}=0$, so that the oscillations in the visibility cannot be traced back to interference among different components. An illustration of the behavior of the visibility in this case has been plotted in Fig. 2, and we always assume, for the sake of generality, a Gaussian distribution of momentum transfers. In this case, the dashed line corresponds to the exponential Markovian decay occurring if only the elastic channel is involved in the dynamics. It immediately appears that a nonmonotonic behavior in the loss of visibility is also observed in this case due to the multiple time integration in Eq. (35).

We have considered the visibility reduction as a function of time. However, in typical interferometric experiments the time of flight is fixed, and it is more natural to study the loss of visibility as a function of the strength of the interaction with the environment. In collisional decohrence, for a fixed time of flight, this depends on the number of collisions, which is directly proportional to the gas density or equivalently to its pressure. As in the Markovian case, we can thus express the visibility as a function of the pressure of the background gas, which is the physical quantity directly tunable in actual experiments [16]. Introducing the effective cross

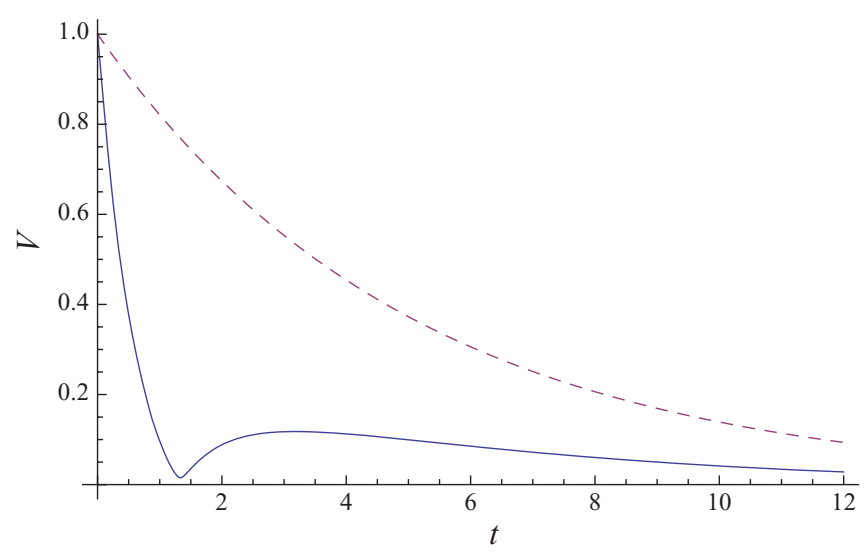

FIG. 2. (Color online) Plot of the visibility in a double-slit arrangement as a function of the interaction time with the environment, in arbitrary units, for the case in which one of the internal states also undergoes inelastic scattering, according to Eq. (47) with $n=2$. It clearly appears a non monotonic decay of the visibility as a consequence of the multiple time integration describing the contribution of the inelastic channel. The distributions of momentum transfers are assumed Gaussian, with $\sigma_{11}=1, \sigma_{12}=3, \mu_{11}=1, \mu_{12}=5$ in a.u.; moreover $\Gamma^{11}=0.75$ and $\Gamma^{12}=1.75$. The dashed line corresponds to the Markovian dynamics determined by the channel undergoing elastic scattering only. 
section $\sigma_{\text {eff }}\left(\boldsymbol{P}_{0}, i\right)$ according to the relation [4]

$$
\sum_{j} \int d \boldsymbol{Q} M_{j j}^{i i}(\boldsymbol{P}+\boldsymbol{Q} ; \boldsymbol{Q})=n_{\mathrm{gas}} \frac{P}{M} \sigma_{\mathrm{eff}}(\boldsymbol{P}, i),
$$

where the lhs denotes the classical loss term appearing in Eq. (18), one has for an ideal gas

$$
\Gamma_{P_{0}}^{i i}=n_{\text {gas }} \frac{P_{0}}{M} \sigma_{\text {eff }}\left(\boldsymbol{P}_{0}, i\right)=\frac{p}{M k_{\mathrm{B}} T} P_{0} \sigma_{\mathrm{eff}}\left(\boldsymbol{P}_{0}, i\right),
$$

where $p$ is the pressure of the gas and $T$ its temperature. One can thus introduce a family of reference pressures depending on the initial internal state of the particle entering the interferometer:

$$
p_{0}^{i}=\frac{M k_{\mathrm{B}} T}{P_{0} \sigma_{\mathrm{eff}}\left(\boldsymbol{P}_{0}, i\right) t},
$$

where $t$ is the time of flight. Then Eq. (43) can equivalently be written as a function of the pressure in the interferometer,

$$
\Gamma_{P_{0}}^{i i} t=\frac{p}{p_{0}^{i}} .
$$

This simply implies that the behavior of the visibility as a function of time is equivalent to its behavior with respect to the pressure and therefore the disturbance of the environment.

\section{CONCLUSIONS}

We have derived the master equation describing the dynamics of a test particle with both translational and internal degrees of freedom, interacting through collisions with a low-density background gas. This was done by building on the so-called monitoring approach [5] and confirms a previous heuristic argument put forward by one of us in [6]. The present microscopic derivation further allows the energy shift to be determined. As we have checked, the result reduces to known equations in suitable limits: the quantum linear Boltzmann equation if the internal degrees of freedom are neglected [4], the master equation for an immobile test particle if the translational degrees of freedom are not relevant $[5,14]$, and quantum classical Markovian master equations if one or both kinds of degrees of freedom can be treated as classical. Note that the natural bases in the derivation were given by momentum for the motional degrees of freedom and energy for the internal ones, the latter corresponding to the channel basis of scattering theory. In these cases, different channels are only coupled through the collision term. If another internal basis can be of interest, coherent tunneling effects also appear.

We have further focused on the situation in which the internal degrees of freedom, in spite of influencing the collisional scattering cross section, are not probed by the measuring apparatus and therefore have to be averaged out from the set of the observed dynamical variables, thus effectively becoming part of the environment. The equation obtained in this situation is no longer of Lindblad type but rather takes the form of a generalized Lindblad structure [6,9]. It can therefore describe behavior quite different from that characterizing a Markovian dynamics. Solving these equations in the position representation for an initial factorized state, we have obtained an explicit expression for the visibility reduction in interferometric experiments when internal degrees of freedom are involved. The behavior of the visibility can indeed be quite different from the exponential decay corresponding to a Markovian dynamics, showing up, for example, as oscillations and revivals.

The interplay between different degrees of freedom in a bipartite system is a natural source of non-Markovian behavior when either of the degrees of freedom cannot be controlled, thus acting as an environment. The scenario here has been studied in a concrete setting, assuming a factorized initial state and describing the dynamics in terms of a generalized Lindblad structure. Such a choice of initial conditions is relevant for the considered interferometric setting; however, it would be of great importance to consider initially correlated states, which naturally appear when considering a non-Markovian dynamics in a strong-coupling regime. It would further be of interest to study whether, instead of using this generalized Lindblad structure, one can obtain a closed description of the reduced system dynamics in terms of a master equation with a memory kernel, at least in some simplified situations. We plan to address these topics in future research work.

\section{ACKNOWLEDGMENTS}

We are grateful to Ludovico Lanz for useful hints in the preparation of the manuscript. BV would also like to thank Klaus Hornberger for very helpful discussions and reading of the manuscript. This work was partially supported by MIUR under PRIN2008.

\section{APPENDIX: MICROSCOPIC DERIVATION OF THE MASTER EQUATION}

Here we address the derivation of the Markovian master equation for the description of the dynamics of the test particle starting from the general expressions of Eqs. (3) and (4). The scattering and rate operators appearing in these equations are best expressed using the factorization of the total Hilbert space, $\mathcal{H}_{\text {tot }}=\mathcal{H}_{\text {sys }} \otimes \mathcal{H}_{\text {gas }}=\mathcal{H}_{\text {c.m. }} \otimes \mathcal{H}_{\text {rel }}$, according to

$$
\begin{aligned}
|\boldsymbol{P}, i\rangle\left\langle\boldsymbol{P}^{\prime}, j|\otimes| \boldsymbol{p}\right\rangle\left\langle\left.\boldsymbol{p}^{\prime}\right|_{\mathrm{gas}}=\right. & |\operatorname{rel}(\boldsymbol{p}, \boldsymbol{P}), i\rangle\left\langle\operatorname{rel}\left(\boldsymbol{p}^{\prime}, \boldsymbol{P}^{\prime}\right),\left.j\right|_{\mathrm{rel}}\right. \\
& \otimes|\boldsymbol{P}+\boldsymbol{p}\rangle\left\langle\boldsymbol{P}^{\prime}+\left.\boldsymbol{p}^{\prime}\right|_{\mathrm{c} . \mathrm{m} .}, \quad(\mathrm{A} 1)\right.
\end{aligned}
$$

where the Hilbert space $\mathbb{C}^{n}$ associated with the internal degrees of freedom is part of $\mathcal{H}_{\text {rel }}$ [27], and the notation is the same as in Sec. II. In fact both operators act in a trivial way on centerof-mass coordinates: $\Gamma=\mathrm{I}_{\mathrm{c} . \mathrm{m} .} \otimes \Gamma_{0}$ and $\mathrm{T}=\mathrm{I}_{\mathrm{c} . \mathrm{m} .} \otimes \mathrm{T}_{0}$. The operator $\Gamma_{0}$ is given by

$$
\Gamma_{0}=\frac{n_{\text {gas }}}{m_{*}} \sum_{j}|\operatorname{rel}(\mathrm{p}, \mathrm{P})| \sigma_{\mathrm{tot}}(\operatorname{rel}(\mathrm{p}, \mathrm{P}), j) \otimes|j\rangle\langle j|,
$$

where $\sigma_{\text {tot }}(\operatorname{rel}(\boldsymbol{p}, \boldsymbol{P}), j)$ is the total cross section depending on the initial relative momentum and internal state. The relation [27]

$$
\left\langle\boldsymbol{p}_{f}, k\left|\mathrm{~T}_{0}\right| \boldsymbol{p}_{i}, j\right\rangle=\frac{1}{2 \pi \hbar m_{*}} \delta\left(\frac{\boldsymbol{p}_{f}^{2}-\boldsymbol{p}_{i}^{2}}{2 m_{*}}+\mathcal{E}_{k j}\right) f_{k j}\left(\boldsymbol{p}_{f}, \boldsymbol{p}_{i}\right)
$$

links the operator $\mathrm{T}_{0}$ to the multichannel complex scattering amplitudes $f_{k j}\left(\boldsymbol{p}_{f}, \boldsymbol{p}_{i}\right)$, referring to scattering from an initial momentum $\boldsymbol{p}_{i}$ and internal state $j$ to a final state 
with momentum $\boldsymbol{p}_{f}$ and internal state $k$. According to standard usage in scattering theory, we call channels the asymptotically free internal energy eigenstates of the system. The differential cross section is given by $\sigma_{k j}\left(\boldsymbol{p}_{f}, \boldsymbol{p}_{i}\right)=$ $\left(\left|\boldsymbol{p}_{f}\right| /\left|\boldsymbol{p}_{i}\right|\right)\left|f_{k j}\left(\boldsymbol{p}_{f}, \boldsymbol{p}_{i}\right)\right|^{2}$, so the total cross section appearing in Eq. (A2) reads $\sigma_{\text {tot }}\left(\boldsymbol{p}_{i}, j\right)=\sum_{k} \int d \boldsymbol{p}_{f} \sigma_{k j}\left(\boldsymbol{p}_{f}, \boldsymbol{p}_{i}\right)$.

\section{Evaluation of the Lindblad structure in momentum and internal state basis \\ a. Incoherent contribution}

We now first concentrate on the evaluation of the contribution given by Eq. (3), which under suitable approximations can be cast in Lindblad form, closely following [12], which dealt with the special case of a test particle without internal structure. To this end, we consider the matrix elements of $\mathcal{L} \rho$ in the momentum and channel basis $\{|\boldsymbol{P}, i\rangle\}$ of the Hilbert space $L^{2}\left(\mathbb{R}^{3}\right) \otimes \mathbb{C}^{n}$ associated with the test particle. Denoting by $\mu(\boldsymbol{p})$ the stationary gas momentum distribution and exploiting Eqs. (A2) and (A3) for the relevant operators, we can express the result as in Eq. (5), where the complex rate functions

$$
\begin{aligned}
M_{i k}^{j l}\left(\boldsymbol{P}, \boldsymbol{P}^{\prime}, \boldsymbol{Q}\right):= & \frac{(2 \pi \hbar)^{3}}{|\Omega|} \int d \boldsymbol{p}_{0} \mu\left(\boldsymbol{p}_{0}\right)\left\langle\operatorname{rel}\left(\boldsymbol{p}_{0}-\boldsymbol{Q}, \boldsymbol{P}\right), i\right| \\
& \times \mathrm{T}_{0} \Gamma_{0}^{1 / 2}\left|\operatorname{rel}\left(\boldsymbol{p}_{0}, \boldsymbol{P}-\boldsymbol{Q}\right), j\right\rangle \\
& \times\left\langle\operatorname{rel}\left(\boldsymbol{p}_{0}, \boldsymbol{P}^{\prime}-\boldsymbol{Q}\right) \mid \Gamma_{0}^{1 / 2} \mathrm{~T}_{0}^{\dagger} \operatorname{rel}\left(\boldsymbol{p}_{0}-\boldsymbol{Q}, \boldsymbol{P}^{\prime}\right)\right\rangle
\end{aligned}
$$

have been introduced, and $|\Omega|$ denotes the volume in which the gas is confined. Note that $M_{i i}^{j j}(\boldsymbol{P}, \boldsymbol{P} ; \boldsymbol{Q})$ can be interpreted as classical rates for scattering of the test particle with momentum $\boldsymbol{P}-\boldsymbol{Q}$ and internal energy eigenstate $j$ to a final state with momentum $\boldsymbol{P}$ and internal energy eigenstate $i$. The contributions on the rhs of Eq. (5) therefore play the role of quantum gain and loss terms, also depending on the internal degrees of freedom involved.

Relying on Eq. (5), we can now deal just with the complex rates $M_{i k}^{j l}\left(\boldsymbol{P}, \boldsymbol{P}^{\prime} ; \boldsymbol{Q}\right)$ defined in Eq. (A4). To proceed, it is helpful to introduce the following functions of $\boldsymbol{p}_{0}$ :

$$
\begin{aligned}
\boldsymbol{p}_{i} & =\operatorname{rel}\left(\boldsymbol{p}_{0}, \frac{\boldsymbol{P}+\boldsymbol{P}^{\prime}}{2}-\boldsymbol{Q}\right), \\
\boldsymbol{p}_{f} & =\operatorname{rel}\left(\boldsymbol{p}_{0}-\boldsymbol{Q}, \frac{\boldsymbol{P}+\boldsymbol{P}^{\prime}}{2}\right),
\end{aligned}
$$

which denote the mean of the pairs of initial and final relative momenta appearing in $M_{i k}^{j l}\left(\boldsymbol{P}, \boldsymbol{P}^{\prime} ; \boldsymbol{Q}\right)$ and which are related by $\boldsymbol{p}_{i}-\boldsymbol{p}_{f}=\boldsymbol{Q}$. Introducing also $\boldsymbol{q}=\operatorname{rel}\left(0,\left(\boldsymbol{P}-\boldsymbol{P}^{\prime}\right) / 2\right)$, the complex functions $M_{i k}^{j l}\left(\boldsymbol{P}, \boldsymbol{P}^{\prime} ; \boldsymbol{Q}\right)$ can be expressed as an average over the gas distribution function $\mu$ of a complex density in the center-of-mass frame

$$
M_{i k}^{j l}\left(\boldsymbol{P}, \boldsymbol{P}^{\prime} ; \boldsymbol{Q}\right)=\int d \boldsymbol{p}_{0} \mu\left(\boldsymbol{p}_{0}\right) m_{i k}^{j l}\left(\boldsymbol{p}_{f}, \boldsymbol{p}_{i} ; \boldsymbol{q}\right)
$$

with

$$
\begin{aligned}
m_{i k}^{j l}\left(\boldsymbol{p}_{f}, \boldsymbol{p}_{i} ; \boldsymbol{q}\right)= & \frac{(2 \pi \hbar)^{3}}{|\Omega|}\left\langle\boldsymbol{p}_{f}+\boldsymbol{q}, i\left|\mathrm{~T}_{0} \Gamma_{0}^{1 / 2}\right| \boldsymbol{p}_{i}+\boldsymbol{q}, j\right\rangle \\
& \times\left\langle\boldsymbol{p}_{i}-\boldsymbol{q}, l\left|\Gamma_{0}^{1 / 2} \mathrm{~T}_{0}^{\dagger}\right| \boldsymbol{p}_{f}-\boldsymbol{q}, k\right\rangle .
\end{aligned}
$$

Evaluating this formula with Eqs. (A2) and (A3) for $\Gamma_{0}$ and $\mathrm{T}_{0}$, respectively, we obtain

$$
\begin{aligned}
m_{i k}^{j l}\left(\boldsymbol{p}_{f}, \boldsymbol{p}_{i} ; \boldsymbol{q}\right) \\
=\frac{2 \pi \hbar}{m_{*}|\Omega|} \Gamma_{0}^{1 / 2}\left(\boldsymbol{p}_{i}+\boldsymbol{q}, j\right) \Gamma_{0}^{1 / 2}\left(\boldsymbol{p}_{i}-\boldsymbol{q}, j^{\prime}\right) \\
\quad \times \delta\left(\frac{\boldsymbol{p}_{f}^{2}-\boldsymbol{p}_{i}^{2}}{2 m_{*}}-\frac{\left(\boldsymbol{p}_{f}-\boldsymbol{p}_{i}\right) \cdot \boldsymbol{q}}{m_{*}}+\mathcal{E}_{k l}\right) \\
\quad \times \delta\left(\frac{\boldsymbol{p}_{f}^{2}-\boldsymbol{p}_{i}^{2}}{2 m_{*}}+\frac{\left(\boldsymbol{p}_{f}-\boldsymbol{p}_{i}\right) \cdot \boldsymbol{q}}{m_{*}}+\mathcal{E}_{i j}\right) \\
\quad \times f_{i j}\left(\boldsymbol{p}_{f}+\boldsymbol{q}, \boldsymbol{p}_{i}+\boldsymbol{q}\right) f_{k l}^{*}\left(\boldsymbol{p}_{f}-\boldsymbol{q}, \boldsymbol{p}_{i}-\boldsymbol{q}\right),
\end{aligned}
$$

where $\Gamma_{0}\left(\boldsymbol{p}_{i}, j\right)=n_{\text {gas }}|\boldsymbol{p}| \sigma_{\text {tot }}(\boldsymbol{p}, j) / m_{*}$ is the eigenvalue of the operator $\Gamma_{0}$ relative to the state $|\boldsymbol{p}, j\rangle$.

The expression given by Eq. (A8) loses its meaning in the infinite-volume limit due to the appearance of the arbitrarily large normalization volume. This point was extensively discussed in [12]. It is to be traced back to the fact that the operator $\Gamma$, in order to provide the actual rate of collisions, should involve a projection on the subspace of incoming wave packets, which is not accounted for in Eq. (A7). To do this, we now evaluate the operator $\Gamma$ on a properly modified state of the relative motion.

Before that, it is convenient to focus our attention on the two $\delta$ functions appearing in Eq. (A8): employing the relation $\delta(a) \delta(b)=2 \delta(a+b) \delta(a-b)$, we can rewrite them as the product

$\frac{1}{2} \delta\left(\frac{\boldsymbol{p}_{f}^{2}-\boldsymbol{p}_{i}^{2}}{2 m_{*}}+\frac{\mathcal{E}_{i j}+\mathcal{E}_{k l}}{2}\right) \delta\left(\frac{\left(\boldsymbol{p}_{f}-\boldsymbol{p}_{i}\right) \cdot \boldsymbol{q}}{m_{*}}+\frac{\mathcal{E}_{i j}-\mathcal{E}_{k l}}{2}\right)$.

These two constraints ensure that the scattering amplitudes appearing in Eq. (A8) are evaluated on shell. The function $m_{i k}^{j l}\left(\boldsymbol{p}_{f}, \boldsymbol{p}_{i} ; \boldsymbol{q}\right)$ gives a significant contribution to the integral in Eq. (A6) when the two energy differences are approximately equal, so that $\mathcal{E}_{i j}=\mathcal{E}_{k l}$, leading otherwise to rapidly oscillating phases. This condition is actually necessary in order to obtain a completely positive time evolution [13]. This implies in particular that, integrating the generalized function $m_{i k}^{j l}\left(\boldsymbol{p}_{f}, \boldsymbol{p}_{i} ; \boldsymbol{q}\right)$ with a function $g(\boldsymbol{q})$, the contributions deriving from the parallel component of $\boldsymbol{q}$ vanish:

$$
m_{i k}^{j l}\left(\boldsymbol{p}_{f}, \boldsymbol{p}_{i} ; \boldsymbol{q}\right) g(\boldsymbol{q})=m_{i k}^{j l}\left(\boldsymbol{p}_{f}, \boldsymbol{p}_{i} ; \boldsymbol{q}_{\perp}\right) g\left(\boldsymbol{q}_{\perp}\right) .
$$

We now therefore evaluate $m_{i k}^{j l}\left(\boldsymbol{p}_{f}, \boldsymbol{p}_{i} ; \boldsymbol{q}_{\perp}\right)$ with a properly modified state of relative motion, which takes into account the restriction of the expression to states that actually describe a colliding pair. To this end, we write the complex rate $m_{i k}^{j l}$ as

$$
\begin{aligned}
m_{i k}^{j l}\left(\boldsymbol{p}_{f}, \boldsymbol{p}_{i} ; \boldsymbol{q}_{\perp}\right) & \\
= & \left\langle\boldsymbol{p}_{f}+\boldsymbol{q}_{\perp}, i\left|\mathrm{~T}_{0} \Gamma_{0}^{1 / 2} \exp \left(i \frac{\mathbf{x}_{\mathrm{rel}} \cdot \boldsymbol{q}_{\perp}}{\hbar}\right) \rho_{\boldsymbol{p}_{i}} \otimes\right| j\right\rangle\langle l| \\
& \times \exp \left(i \frac{\mathbf{x}_{\mathrm{rel}} \cdot \boldsymbol{q}_{\perp}}{\hbar}\right) \Gamma_{0}^{1 / 2} \mathrm{~T}_{0}^{\dagger}\left|\boldsymbol{p}_{f}-\boldsymbol{q}_{\perp}, k\right\rangle,
\end{aligned}
$$

where $\rho_{\boldsymbol{p}_{i}}$ denotes an improper state of relative motion,

$$
\rho_{\boldsymbol{p}_{i}}=\frac{(2 \pi \hbar)^{3}}{|\Omega|}\left|\boldsymbol{p}_{i}\right\rangle\left\langle\boldsymbol{p}_{i}\right|
$$


Since the rate operator $\Gamma$ should have a vanishing expectation value for those states of the relative motion that are not of the incoming type, we make the replacement

$$
\begin{aligned}
\rho_{\boldsymbol{p}_{i}} \otimes|j\rangle\left\langle l\left|\rightarrow \rho_{\boldsymbol{p}_{i}}^{\prime} \otimes\right| j\right\rangle\langle l| \\
=\int_{\Lambda_{\boldsymbol{p}_{i}}} \frac{d \boldsymbol{x}_{\| \boldsymbol{p}_{i}}}{\left|\Lambda_{\boldsymbol{p}_{i}}\right|} \int_{\Sigma_{\boldsymbol{p}_{i}}} \frac{d \boldsymbol{x}_{\perp \boldsymbol{p}_{i}}}{\left|\Sigma_{\boldsymbol{p}_{i}}\right|} \int d \boldsymbol{w} e^{i \boldsymbol{x} \cdot \boldsymbol{w} / \hbar} \\
\quad \times\left|\boldsymbol{p}_{i}-\frac{\boldsymbol{w}}{2}, j\right\rangle\left\langle\boldsymbol{p}_{i}+\frac{\boldsymbol{w}}{2}, l\right| .
\end{aligned}
$$

This corresponds to a restriction of the Wigner function associated with the improper state of relative motion [Eq. (A10)] from the entire normalization volume $|\Omega|$ to a cylinder pointing in the direction $\boldsymbol{p}_{i}$, with base surface $\Sigma_{\boldsymbol{p}_{i}}$ and height $\Lambda_{p_{i}}$. As in the case without internal degrees of freedom, $\Lambda_{p_{i}}$ is approximately the distance traveled by the particle between two subsequent collisions, while $\Sigma_{\boldsymbol{p}_{i}}$ can now be taken as the geometric mean of the total cross section of the involved channels [5], that is to say $\left|\Sigma_{\boldsymbol{p}_{i}}\right|=\sqrt{\sigma\left(\boldsymbol{p}_{i}, j\right) \sigma\left(\boldsymbol{p}_{i}, l\right)}$ and $\left|\Lambda_{p_{i}}\right|=\left(\left|\boldsymbol{p}_{i}\right| \Delta t\right) / m_{*}$, with $\Delta t$ the typical time interval between two subsequent collisions.

Putting the new state, Eq. (A11), into Eq. (A7) and using the expressions of the matrix elements of $T_{0}$ and $\Gamma_{0}$, we get

$$
\begin{aligned}
m_{i k}^{j l}\left(\boldsymbol{p}_{f}, \boldsymbol{p}_{i} ; \boldsymbol{q}_{\perp}\right) \\
=\int_{\Lambda_{p_{i}}} \frac{d \boldsymbol{x}_{\| \boldsymbol{p}_{i}}}{\left|\Lambda_{\boldsymbol{p}_{i}}\right|} \int_{\Sigma_{\boldsymbol{p}_{i}}} \frac{d \boldsymbol{x}_{\perp \boldsymbol{p}_{i}}}{\left|\Sigma_{\boldsymbol{p}_{i}}\right|} \int d \boldsymbol{w} \exp \left(-i \frac{\boldsymbol{x} \cdot \boldsymbol{w}}{\hbar}\right) \frac{1}{\left(2 \pi \hbar m_{*}\right)^{2}} \\
\quad \times \frac{n_{\mathrm{gas}}}{\left|\boldsymbol{p}_{i}\right|} \delta\left(\frac{\boldsymbol{p}_{f}^{2}-\boldsymbol{p}_{i}^{2}}{2 m_{*}}-\frac{\boldsymbol{w}^{2}}{8 m_{*}}-\frac{\boldsymbol{q}_{\perp} \cdot \boldsymbol{w}}{2 m_{*}}+\frac{\mathcal{E}_{i j}+\mathcal{E}_{k l}}{2}\right) \\
\quad \times \delta\left(\frac{\boldsymbol{p}_{i} \cdot \boldsymbol{w}}{\left|\boldsymbol{p}_{i}\right|}+\frac{m_{*}}{\left|\boldsymbol{p}_{i}\right|}\left(\mathcal{E}_{k l}-\mathcal{E}_{i j}\right)\right) \\
\quad \times f_{i j}\left(\boldsymbol{p}_{f}+\boldsymbol{q}_{\perp}, \boldsymbol{p}_{i}+\boldsymbol{q}_{\perp}+\frac{\boldsymbol{w}}{2}\right) \\
\quad \times f_{k l}^{*}\left(\boldsymbol{p}_{f}-\boldsymbol{q}_{\perp}, \boldsymbol{p}_{i}-\boldsymbol{q}_{\perp}-\frac{\boldsymbol{w}}{2}\right) \\
\quad \times \sqrt{\left|\boldsymbol{p}_{i}+\boldsymbol{q}_{\perp}+\frac{\boldsymbol{w}}{2} \| \boldsymbol{p}_{i}-\boldsymbol{q}_{\perp}-\frac{\boldsymbol{w}}{2}\right|} \\
\quad \times \sqrt{\sigma\left(\boldsymbol{p}_{i}+\boldsymbol{q}_{\perp}+\frac{\boldsymbol{w}}{2}, j\right) \sigma\left(\boldsymbol{p}_{i}-\boldsymbol{q}_{\perp}-\frac{\boldsymbol{w}}{2}, l\right)}
\end{aligned}
$$

where we have once again exploited the relation $\delta(a) \delta(b)=$ $2 \delta(a+b) \delta(a-b)$. We now first perform the integral over $\boldsymbol{w}_{\| \boldsymbol{p}_{i}}$, which denotes the component of $\boldsymbol{w}$ parallel to $\boldsymbol{p}_{i}$, thus evaluating the second $\delta$ function on the rhs of the previous equation, so that the dependence on $\boldsymbol{x}_{\| p_{i}}$ only appears in the term

$$
\int_{\Lambda_{p_{i}}} \frac{d \boldsymbol{x}_{\| \boldsymbol{p}_{i}}}{\left|\Lambda_{\boldsymbol{p}_{i} \mid}\right|} \exp \left[-\frac{i}{\hbar} \boldsymbol{x}_{\| \boldsymbol{p}_{i}} \cdot\left(\frac{m_{*}}{\left|\boldsymbol{p}_{i}\right|}\left(\mathcal{E}_{i j}-\mathcal{E}_{k l}\right)\right) \hat{\boldsymbol{p}}_{i}\right],
$$

where the hat symbol denotes the versor. The phase of the integrand varies very quickly for $\left(\mathcal{E}_{i j}-\mathcal{E}_{k l}\right) \gg \hbar / \Delta t$, where $\Delta t$ is the typical time elapsing between collisions, so that, as already discussed, its contribution vanishes unless $\mathcal{E}_{i j}=\mathcal{E}_{k l}$, corresponding to a rotating-wave approximation, assuming a separation of time scales between internal and translational dynamics [3]. Further considering the integral over $\boldsymbol{x}_{\perp \boldsymbol{p}_{i}}$ as an approximate expression for $\delta\left(\boldsymbol{w}_{\perp \boldsymbol{p}_{i}}\right)$, we are led to

$$
\begin{aligned}
m_{i k}^{j l}\left(\boldsymbol{p}_{f}, \boldsymbol{p}_{i} ; \boldsymbol{q}_{\perp}\right) \\
=\frac{n_{\mathrm{gas}}}{m_{*}^{2}} \chi_{i k}^{j l} f_{i j}\left(\boldsymbol{p}_{f}+\boldsymbol{q}_{\perp}, \boldsymbol{p}_{i}+\boldsymbol{q}_{\perp}\right) \\
\quad \times f_{k l}^{*}\left(\boldsymbol{p}_{f}-\boldsymbol{q}_{\perp}, \boldsymbol{p}_{i}-\boldsymbol{q}_{\perp}\right) \delta\left(\frac{\boldsymbol{p}_{f}^{2}-\boldsymbol{p}_{i}^{2}}{2 m_{*}}+\mathcal{E}_{i j}\right) \\
\quad \times \frac{\sqrt{\left|\boldsymbol{p}_{i}+\boldsymbol{q}_{\perp}\right|\left|\boldsymbol{p}_{i}-\boldsymbol{q}_{\perp}\right|}}{\left|\boldsymbol{p}_{i}\right|} \frac{\sqrt{\sigma\left(\boldsymbol{p}_{i}+\boldsymbol{q}_{\perp}, j\right) \sigma\left(\boldsymbol{p}_{i}-\boldsymbol{q}_{\perp}, l\right)}}{\sqrt{\sigma\left(\boldsymbol{p}_{i}, j\right) \sigma\left(\boldsymbol{p}_{i}, l\right)}},
\end{aligned}
$$

where the $\chi_{i k}^{j l}$ act like a Kronecker's delta factor, defined according to

$$
\chi_{i k}^{j l}= \begin{cases}1 & \text { if } \mathcal{E}_{i j}=\mathcal{E}_{k l}, \\ 0 & \text { otherwise. }\end{cases}
$$

In the last two terms, we can disregard the dependence on $\boldsymbol{q}_{\perp}$ because we expect that a $\boldsymbol{q}_{\perp}$ integration will average out the "far off-diagonal" contributions with large modulus $\left|\boldsymbol{q}_{\perp}\right|$, where the phases of the two scattering amplitudes are no longer synchronous. In conclusion, we have

$$
\begin{aligned}
& m_{i k}^{j l}\left(\boldsymbol{p}_{f}, \boldsymbol{p}_{i} ; \boldsymbol{q}_{\perp}\right) \\
& \quad=\frac{n_{\mathrm{gas}}}{m_{*}^{2}} \chi_{i k}^{j l} \delta\left(\frac{\boldsymbol{p}_{f}^{2}-\boldsymbol{p}_{i}^{2}}{2 m_{*}}+E_{i}-E_{j}\right) \\
& \times f_{i j}\left(\boldsymbol{p}_{f}+\boldsymbol{q}_{\perp}, \boldsymbol{p}_{i}+\boldsymbol{q}_{\perp}\right) f_{k l}^{*}\left(\boldsymbol{p}_{f}-\boldsymbol{q}_{\perp}, \boldsymbol{p}_{i}-\boldsymbol{q}_{\perp}\right) .
\end{aligned}
$$

This relation determines the complex rate functions $M_{i k}^{j l}\left(\boldsymbol{P}, \boldsymbol{P}^{\prime} ; \boldsymbol{Q}\right)$ through Eq. (A6) and, therefore, the dissipative part of the master equation according to Eq. (5).

\section{b. Energy shift}

As a last step in the determination of the structure of the master equation, we need to evaluate the contribution given by $\mathcal{R} \rho$. In the same notation as above, and within the same approximations, we directly obtain

$$
\begin{aligned}
& \left\langle\boldsymbol{P}, i|\mathcal{R} \rho| \boldsymbol{P}^{\prime}, k\right\rangle \\
& =\left\langle\boldsymbol{P}, i\left|i \operatorname{Tr}_{\mathrm{gas}}\left\{\left[\Gamma^{1 / 2} \operatorname{Re}(\mathrm{T}) \Gamma^{1 / 2}, \rho \otimes \rho_{\mathrm{gas}}\right]\right\}\right| \boldsymbol{P}^{\prime}, k\right\rangle \\
& =\frac{1}{i \hbar} \sum_{j}\left(E_{n}^{i j}(\boldsymbol{P})\left\langle\boldsymbol{P}, j|\rho| \boldsymbol{P}^{\prime}, k\right\rangle-E_{n}^{j k}\left(\boldsymbol{P}^{\prime}\right)\left\langle\boldsymbol{P}, i|\rho| \boldsymbol{P}^{\prime}, j\right\rangle\right),
\end{aligned}
$$

with

$$
\begin{aligned}
E_{n}^{i j}(\boldsymbol{P})= & -2 \pi \hbar^{2} \frac{n_{\text {gas }}}{m_{*}} \chi_{i k}^{j k} \int d \boldsymbol{p}_{0} \mu\left(\boldsymbol{p}_{0}\right) \\
& \times \operatorname{Re}\left[f_{i j}\left(\operatorname{rel}\left(\boldsymbol{p}_{0}, \boldsymbol{P}\right), \operatorname{rel}\left(\boldsymbol{p}_{0}, \boldsymbol{P}\right)\right)\right] .
\end{aligned}
$$

It is worth noting that, for the case of a nondegenerate free internal Hamiltonian, this formula reduces to

$$
\left\langle\boldsymbol{P}, i|\mathcal{R} \rho| \boldsymbol{P}^{\prime}, k\right\rangle=\frac{1}{i \hbar}\left(E_{n}^{i}(\boldsymbol{P})-E_{n}^{k}\left(\boldsymbol{P}^{\prime}\right)\right)\left\langle\boldsymbol{P}, i|\rho| \boldsymbol{P}^{\prime}, k\right\rangle
$$

with

$$
\begin{aligned}
E_{n}^{i}(\boldsymbol{P})= & -2 \pi \hbar^{2} \frac{n_{\mathrm{gas}}}{m_{*}} \int d \boldsymbol{p}_{0} \mu\left(\boldsymbol{p}_{0}\right) \\
& \times \operatorname{Re}\left[f_{i i}\left(\operatorname{rel}\left(\boldsymbol{p}_{0}, \boldsymbol{P}\right), \operatorname{rel}\left(\boldsymbol{p}_{0}, \boldsymbol{P}\right)\right)\right] .
\end{aligned}
$$




\section{A. Operator expression of the master equation}

We now recast the master equation, Eq. (1), whose matrix elements are given by Eqs. (5) and (A13), in a way that allows us to express it in a representation-independent form. The key point is to show that $M_{i k}^{j l}\left(\boldsymbol{P}, \boldsymbol{P}^{\prime} ; \boldsymbol{Q}\right)=$ $\int d \boldsymbol{p}_{0} \mu\left(\boldsymbol{p}_{0}\right) m_{i k}^{j l}\left(\boldsymbol{p}_{f}, \boldsymbol{p}_{i} ; \boldsymbol{q}\right)$ can be factorized into two terms, one depending on $\boldsymbol{P}$ and the other on $\boldsymbol{P}^{\prime}$.

Changing the integration variable from $\boldsymbol{p}_{0}$ to $\boldsymbol{p}_{i}$ and using Eq. (A5) to obtain $\boldsymbol{p}_{0}=\boldsymbol{p}_{i}+\left(\boldsymbol{p}_{f}+\boldsymbol{P}\right) m / M+\boldsymbol{q} m / m_{*}=$ $\boldsymbol{p}_{i}+\left(\boldsymbol{p}_{f}+\boldsymbol{P}^{\prime}\right) m / M-\boldsymbol{q} m / m_{*}$, we have

$$
\begin{aligned}
M_{i k}^{j l} & \left(\boldsymbol{P}, \boldsymbol{P}^{\prime} ; \boldsymbol{Q}\right) \\
= & \frac{m^{3}}{m_{*}^{3}} \frac{n_{\mathrm{gas}}}{m_{*}} \chi_{i k}^{j l} \int d \boldsymbol{p}_{i} \delta\left(\frac{\boldsymbol{p}_{f}^{2}-\boldsymbol{p}_{i}^{2}}{2 m_{*}}+\mathcal{E}_{i j}\right) \\
& \times \mu^{1 / 2}\left(\boldsymbol{p}_{i}+\frac{m}{M}\left(\boldsymbol{p}_{f}+\boldsymbol{P}\right)+\frac{m}{m_{*}} \boldsymbol{q}_{\perp}\right) \\
& \times \mu^{1 / 2}\left(\boldsymbol{p}_{i}+\frac{m}{M}\left(\boldsymbol{p}_{f}+\boldsymbol{P}^{\prime}\right)-\frac{m}{m_{*}} \boldsymbol{q}_{\perp}\right) \\
& \times f_{i j}\left(\boldsymbol{p}_{f}+\boldsymbol{q}_{\perp}, \boldsymbol{p}_{i}+\boldsymbol{q}_{\perp}\right) f_{k l}^{*}\left(\boldsymbol{p}_{f}-\boldsymbol{q}_{\perp}, \boldsymbol{p}_{i}-\boldsymbol{q}_{\perp}\right),
\end{aligned}
$$

where we replaced $\boldsymbol{q}$ by $\boldsymbol{q}_{\perp}$ in the arguments of $\mu^{1 / 2}$, in accordance with Eq. (A9). Remembering that $\boldsymbol{p}_{i}-\boldsymbol{p}_{f}=$ $\boldsymbol{Q}$ and $\boldsymbol{q}=m_{*}\left(\boldsymbol{P}-\boldsymbol{P}^{\prime}\right) /(2 M)$, we consider the change of variable

$$
\boldsymbol{p}_{i} \rightarrow \frac{m}{m_{*}} \boldsymbol{p}_{i}+\frac{m}{M} \frac{\boldsymbol{P}_{\perp}+\boldsymbol{P}_{\perp}^{\prime}}{2}-\frac{m}{m_{*}} \frac{\boldsymbol{Q}}{2}-\frac{\mathcal{E}_{i j}}{Q^{2} / m} \boldsymbol{Q}
$$

to obtain the desired factorization. If we further consider that the $\delta$ function $\delta(\boldsymbol{p} \cdot \boldsymbol{Q} / \mathrm{m})$ restricts the $\boldsymbol{p}$ integration to the plane $\boldsymbol{Q}_{\perp}=\left\{\boldsymbol{p} \in \mathbb{R}^{3}: \boldsymbol{p} \cdot \boldsymbol{Q}=0\right\}$, we finally arrive at Eq. (6), with $L_{i j}(\boldsymbol{p}, \boldsymbol{P} ; \boldsymbol{Q})$ as in Eq. (7), which allows us to obtain the operator expression of the master equation given by Eqs. (9) and (10). If the gas distribution function $\mu$ is given by a Maxwell-Boltzmann probability density $\mu_{\beta}(\boldsymbol{p})=1 /\left(\pi^{3 / 2} p_{\beta}^{3}\right) \exp \left(-\boldsymbol{p}^{2} / p_{\beta}^{2}\right)$, where $p_{\beta}=\sqrt{2 m / \beta}$ is the most probable momentum at temperature $T=1 /\left(k_{B} \beta\right)$, these functions can be expressed in terms of the dynamic structure factor for a Maxwell-Boltzmann gas [28,29],

$$
S_{\mathrm{MB}}(\boldsymbol{Q}, E)=\sqrt{\frac{\beta m}{2 \pi}} \frac{1}{Q} \exp \left(-\frac{\beta}{8 m} \frac{\left(Q^{2}+2 m E\right)^{2}}{Q^{2}}\right) .
$$

In fact, using the relation

$$
\begin{aligned}
& \frac{m}{Q} \mu_{\beta}\left(\boldsymbol{p}_{\perp}+\frac{m}{M} \boldsymbol{P}_{\|}+\left(1+\frac{m}{M}\right) \frac{\boldsymbol{Q}}{2}+\frac{\mathcal{E}_{i j}}{Q^{2} / m} \boldsymbol{Q}\right) \\
& =\mu_{\beta}\left(\boldsymbol{p}_{\perp}\right) S_{\mathrm{MB}}\left(\boldsymbol{Q}, E(\boldsymbol{Q}, \boldsymbol{P})+\mathcal{E}_{i j}\right),
\end{aligned}
$$

with $E(\boldsymbol{Q}, \boldsymbol{P})=:(\boldsymbol{P}+\boldsymbol{Q})^{2} / 2 M-\boldsymbol{P}^{2} / 2 M$, the energy transferred to the center of mass in a collision changing the momentum of the test particle from $\boldsymbol{P}$ to $\boldsymbol{P}+\boldsymbol{Q}$, Eq. (7) can be written as [6]

$$
\begin{aligned}
L_{i j}(\boldsymbol{p}, \boldsymbol{P} ; \boldsymbol{Q})= & \sqrt{\frac{n_{\mathrm{gas}}}{m_{*}^{2}} \mu_{\beta}\left(\boldsymbol{p}_{\perp}\right)} \sqrt{S_{\mathrm{MB}}\left(\boldsymbol{Q}, E(\boldsymbol{Q}, \boldsymbol{P})+\mathcal{E}_{i j}\right)} \\
& \times f_{i j}\left(\operatorname{rel}\left(\boldsymbol{p}_{\perp}, \boldsymbol{P}_{\perp}\right)-\frac{\boldsymbol{Q}}{2}+\frac{\mathcal{E}_{i j}}{Q^{2} / m_{*}} \boldsymbol{Q},\right. \\
& \left.\operatorname{rel}\left(\boldsymbol{p}_{\perp}, \boldsymbol{P}_{\perp}\right)+\frac{\boldsymbol{Q}}{2}+\frac{\mathcal{E}_{i j}}{Q^{2} / m_{*}} \boldsymbol{Q}\right) .
\end{aligned}
$$

In the latter expression for the Lindblad operators, the dynamic structure factor appears to be evaluated for an energy transfer corresponding to the sum of the contributions for center of mass and internal state, as naturally expected. As discussed in [4], the dynamic structure factor describes momentum and energy transferred to the test particle when scattering off a macroscopic system, thus allowing for a more transparent physical understanding of the structure of the Lindblad operators.
[1] E. Joos, H. D. Zeh, C. Kiefer, D. Giulini, J. Kupsch, and I.-O. Stamatescu, Decoherence and the Appearance of a Classical World in Quantum Theory, 2nd ed. (Springer, Berlin, 2003).

[2] M. Schlosshauer, Decoherence and the Quantum-to-Classical Transition (Springer-Verlag, Berlin, 2007).

[3] H.-P. Breuer and F. Petruccione, The Theory of Open Quantum Systems (Oxford University, Oxford, 2007).

[4] B. Vacchini and K. Hornberger, Phys. Rep. 478, 71 (2009).

[5] K. Hornberger, Europhys. Lett. 77, 50007 (2007).

[6] B. Vacchini, Phys. Rev. A 78, 022112 (2008).

[7] S. Kryszewski and J. Czechowska, Phys. Rev. A 74, 022719 (2006).

[8] A. A. Budini, Phys. Rev. A 74, 053815 (2006).

[9] H.-P. Breuer, Phys. Rev. A 75, 022103 (2007).

[10] K. Hornberger, Phys. Rev. Lett. 97, 060601 (2006).

[11] K. Hornberger, J. Phys.: Conf. Ser. 67, 012002 (2007).

[12] K. Hornberger and B. Vacchini, Phys. Rev. A 77, 022112 (2008).
[13] R. Alicki and S. Kryszewski, Phys. Rev. A 68, 013809 (2003).

[14] R. Dümcke, Commun. Math. Phys. 97, 331 (1985).

[15] R. F. Snider, Int. Rev. Phys. Chem. 17, 185 (1998).

[16] K. Hornberger, S. Uttenthaler, B. Brezger, L. Hackermüller, M. Arndt, and A. Zeilinger, Phys. Rev. Lett. 90, 160401 (2003).

[17] C. Champenois, M. Jacquey, S. Lepoutre, M. Buchner, G. Trenec, and J. Vigue, Phys. Rev. A 77, 013621 (2008).

[18] M. Jacquey, M. Buchner, G. Trenec, and J. Vigue, Phys. Rev. Lett. 98, 240405 (2007).

[19] M. Hillery, L. Mlodinow, and V. Buzek, Phys. Rev. A 71, 062103 (2005)

[20] C. J. Hemming and R. V. Krems, Phys. Rev. A 81, 052701 (2010).

[21] W. Feller, An Introduction to Probability Theory and Its Applications (Wiley, New York, 1971), vol. II.

[22] C. M. Savage and D. F. Walls, Phys. Rev. A 32, 2316 (1985).

[23] K. Hornberger, Phys. Rev. A 73, 052102 (2006).

[24] B. Vacchini in Theoretical Foundations of Quantum Information Processing and Communication, edited by E. Bruening and 
F. Petruccione, Lecture Notes in Physics, vol. 787 (Springer, Berlin, 2010).

[25] P. A. Alemany, J. Phys. A 30, 6587 (1997).

[26] D. A. Kokorowski, A. D. Cronin, T. D. Roberts, and D. E. Pritchard, Phys. Rev. Lett. 86, 2191 (2001).
[27] J. R. Taylor, Scattering Theory (J. Wiley \& Sons, New York, 1972).

[28] F. Schwabl, Advanced Quantum Mechanics (Springer, New York, 2003).

[29] L. Pitaevskii and S. Stringari, Bose-Einstein Condensation (Oxford University, Oxford, 2003). 